Supporting Information

\title{
Metal-Free Photocatalyzed Cross Coupling of Bromo-Heteroarenes with Pyrroles
}

\author{
Leyre Marzo, ${ }^{\dagger}$ Indrajit Ghosh, ${ }^{\dagger}$ Francisco Esteban, ${ }^{\ddagger}$ and Burkhard König*† \\ †Institute of Organic Chemistry, University of Regensburg, D-93040 Regensburg, \\ Germany. \\ ‡Department of Organic Chemistry (Modulo I), Universidad Autónoma de \\ Madrid, Cantoblanco, 28049, Madrid, Spain. \\ E-mail: Burkhard.Koenig@.ur.de.
}

Table of Contents

1. General Methods and Materials 


\section{General Methods and Materials:}

All NMR spectra were recorded at room temperature using a Bruker Avance 300 (300 MHz for $1 \mathrm{H}, 75 \mathrm{MHz}$ for $13 \mathrm{C}$ ), or a Bruker Avance 400 (400 MHz for $1 \mathrm{H}, 101 \mathrm{MHz}$ for 13C) NMR spectrometer. All chemical shifts are reported in $\delta$-scale as parts per million [ppm] (multiplicity, coupling constant $J$, number of protons) relative to the solvent residual peaks as the internal standard. ${ }^{1}$ Coupling constants $J$ are given in Hertz $[\mathrm{Hz}]$. Abbreviations used for signal multiplicity: $1 \mathrm{H}-\mathrm{NMR}: \mathrm{b}=$ broad, $\mathrm{s}=$ singlet, $\mathrm{d}=$ doublet, $\mathrm{t}=$ triplet, $\mathrm{q}=$ quartet, $\mathrm{dd}=$ doublet of doublets, $\mathrm{dt}=$ doublet of triplets, and $\mathrm{m}=$ multiplet. The mass spectrometric measurements were performed at the Central Analytical Laboratory of the University of Regensburg. All mass spectra were recorded on a Finnigan MAT 95, Thermo Quest Finnigan TSQ 7000, Finnigan MATSSQ 710 A or an Agilent Q-TOF 6540 UHD instrument. GC measurements were performed on a GC 7890 from Agilent Technologies. Data acquisition and evaluation was done with Agilent ChemStation Rev.C.01.04. [35]. GC/MS measurements were performed on a 7890A GC system from Agilent Technologies with an Agilent 5975MSD Detector. Data acquisition and evaluation was done with MSD ChemStationE.02.02.1431.A capillary column HP-5MS/30 $\mathrm{m} \times 0.25 \mathrm{~mm} / 0.25 \mu \mathrm{M}$ film and helium as carrier gas (flow rate of $1 \mathrm{~mL} / \mathrm{min}$ ) were used. The injector temperature (split injection: 40:1 split) was $280{ }^{\circ} \mathrm{C}$, detection temperature $300{ }^{\circ} \mathrm{C}$ (FID). GC measurements were made and investigated via integration of the signal obtained. The GC oven temperature program was adjusted as follows: initial temperature $40{ }^{\circ} \mathrm{C}$ was kept for 3 minutes, the temperature was increased at a rate of $15{ }^{\circ} \mathrm{C} / \mathrm{min}$ over a period of 16 minutes until $280^{\circ} \mathrm{C}$ was reached and kept for 5 minutes, the temperature was again increased at a rate of $25^{\circ} \mathrm{C} / \mathrm{min}$ over a period of 48 seconds until the final temperature $\left(300^{\circ} \mathrm{C}\right)$ was reached and kept for 5 minutes. Analytical TLC was performed on silica gel coated alumina plates (MN TLC sheets ALUGRAM $^{\circledR}$ Xtra SIL G/UV254).Visualization was done by UV light ( 254 or $366 \mathrm{~nm}$ ). If necessary, potassium permanganate was used for chemical staining. Purification by column chromatography was performed with silica gel $60 \mathrm{M}$ (40-63 $\mu \mathrm{m}, 230-440$ mesh, Merck) on a Biotage ${ }^{\circledR}$ Isolera TM Spektra One device. C-H arylation reactions were performed with $455 \mathrm{~nm}$ LEDs (OSRAM Oslon SSL 80 royal-blue LEDs ( $\lambda=455 \mathrm{~nm}$ $( \pm 15 \mathrm{~nm}), 3.5 \mathrm{~V}, 700 \mathrm{~mA})$. UV-Vis and fluorescence measurements were performed with a Varian Cary 50 UV/Vis spectrophotometer and FluoroMax-4 spectrofluorometer, respectively. Commercially available starting materials and solvents were used without further purification. 


\section{Experimental Procedures and Characterizations:}

General procedure A: Reaction in DMSO. In a $5 \mathrm{~mL}$ snap vial with magnetic stirring bar the heteroaryl halide $\left(0.1 \mathrm{mmol}, 1\right.$ equiv), the catalyst Rh-6G $(0.01 \mathrm{mmol}, 0.1 \text { equiv. })^{2}$ and the corresponding pyrrole ( $1.8 \mathrm{mmol}, 18$ equiv.) under $\mathrm{N}_{2}$ atmosphere were dissolved in dry DMSO $(1.5 \mathrm{~mL})$ and the resulting mixture was degassed under vacuum via syringe needle. DIPEA $(0.15$ mmol, 1.5 equiv.) was added under $\mathrm{N}_{2}$ and the reaction mixture was degassed (x2) under vacuum via syringe needle. The snap capped vial containing the final reaction mixture was placed in a cooling block (see Figure S1) and the mixture was irradiated through the plane bottom side of the snap vial using a $455 \mathrm{~nm}$ LED. The reaction progress was monitored by GC analysis. For work up, the reaction mixture was transferred into a separating funnel and $10 \mathrm{~mL}$ of distilled water and $2 \mathrm{~mL}$ of brine were added. The resulting mixture was extracted with ethyl acetate $(3 \times 10 \mathrm{~mL})$. The combined organic layers were dried over $\mathrm{MgSO}_{4}$, filtered and concentrated in vacuum. Purification of the crude product was carried out by flash column chromatography using petrol ether/ethyl acetate or pentane/Et ${ }_{2} \mathrm{O}$ as indicated in each case.

General procedure B: Reaction in ACN. In a $5 \mathrm{~mL}$ snap vial with magnetic stirring bar the heteroaryl halide $\left(0.1 \mathrm{mmol}, 1\right.$ equiv.), the catalyst $\mathrm{Rh}-6 \mathrm{G}(0.01 \mathrm{mmol}, 0.1 \text { equiv. })^{2}$ and the corresponding pyrrole ( $1.8 \mathrm{mmol}, 18$ equiv.) under $\mathrm{N}_{2}$ atmospherewere dissolved in dry ACN $(1.5 \mathrm{~mL})$ and DIPEA $(0.15 \mathrm{mmol}, 1.5$ equiv.) was added to the solution. The mixture was cooled down to $-78 \stackrel{\circ}{\circ}$ and degassed under vacuum via syringe needle for two times. The snap capped vial containing the final reaction mixture was placed in a cooling block (see Figure S1) and the mixture was irradiated through the plane bottom side of the snap vial using a $455 \mathrm{~nm}$ LED. The reaction progress was monitored by GC analysis. The solvent of the reaction is removed under vacuum and the product purified by flash column chromatography using petrol ether/ethyl acetate or pentane $/ \mathrm{Et}_{2} \mathrm{O}$ as indicated in each case.

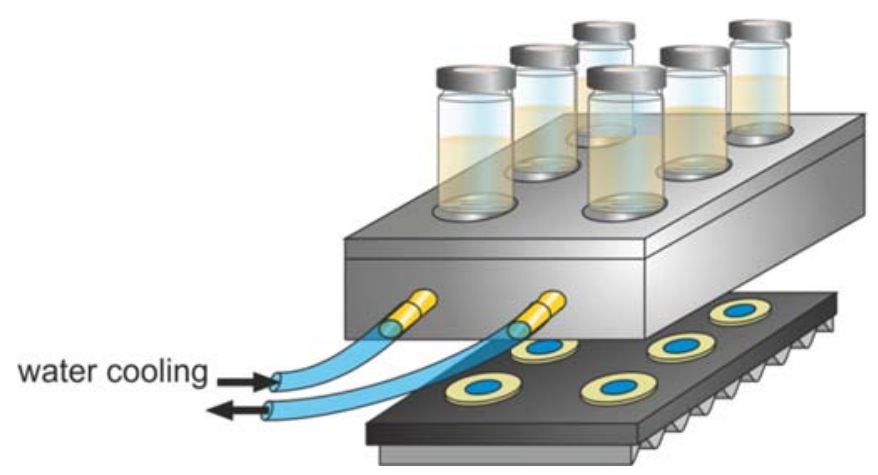

Figure S1: Schematic representation of the photochemical reaction setup.

\section{Characterization of isolated compounds}

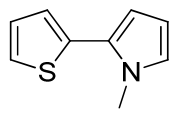

1-Methyl-2-(thiophen-2-yl)-1H-pyrrole (3a):The product was obtained following the general procedure $B$, using 20 mol\% catalyst (Rh-6G 0.02 mmol, 0.2 equiv.) in $48 \mathrm{~h}$ of reaction, as a colorless oil in $65 \%$ yield. Once the reaction is finished, the crude mixture is absorbed on silica gel, the solvent removed under vacuum, and the product purified by flash chromatography (eluent pentane). The experimental data agree with the data previously described in the literature. ${ }^{3}{ }^{1} \mathrm{H}-\mathrm{NMR}\left(300 \mathrm{MHz}, \mathrm{CDCl}_{3}\right) \delta: 7.26(\mathrm{dd}, J=5.1,1.2 \mathrm{~Hz}$, $1 \mathrm{H}), 7.10-7.04(\mathrm{~m}, 1 \mathrm{H}), 7.02(\mathrm{dd}, J=3.6,1.2 \mathrm{~Hz}, 1 \mathrm{H}), 6.74-6.68(\mathrm{~m}, 1 \mathrm{H}), 6.33(\mathrm{dd}, J=3.6,1.7$ $\mathrm{Hz}, 1 \mathrm{H}), 6.17(\mathrm{dd}, J=3.6,2.7 \mathrm{~Hz}, 1 \mathrm{H}), 3.72(\mathrm{~s}, 3 \mathrm{H}) . \mathrm{HMRS}$ : [M+] calculated for $\mathrm{C}_{9} \mathrm{H}_{9} \mathrm{SN}$ : 163.0450;found: 163.0449 . 
2-(Thiophen-2-yl)-1H-pyrrole (3b): The product was obtained following the general procedure A using 20 mol\% catalyst (Rh-6G 0.02 mmol, 0.2 equiv.) in $48 \mathrm{~h}$ of reaction, as a colorless oil in $41 \%$ yield. After the workup, the crude product is adsorbed on silica gel, the solvent evaporated under vacuum, and the product purified by flash chromatography (eluent 90/10, pentane/Et ${ }_{2} \mathrm{O}$ ). ${ }^{1} \mathrm{H}-\mathrm{NMR}\left(300 \mathrm{MHz}, \mathrm{CDCl}_{3}\right) \delta: 8.31$ (bs, $1 \mathrm{H}$ ), 7.15 $(\mathrm{dd}, J=3.8,1.6 \mathrm{~Hz}, 1 \mathrm{H}), 7.04-7.01(\mathrm{~m}, 2 \mathrm{H}), 6.82-6.80(\mathrm{~m}, 1 \mathrm{H}), 6.43-6.41(\mathrm{~m}, 1 \mathrm{H}), 6.27(\mathrm{dd}, J=$ 6.1, 2.6 Hz, 1H). ${ }^{13} \mathrm{C}-\mathrm{NMR}\left(300 \mathrm{MHz}, \mathrm{CDCl}_{3}\right.$ ) $\delta: 136.3,127.6,126.7,122.7,120.9,118.5,110.0$, 106.7. $\mathrm{HMRS}:\left[\mathrm{M}^{+}\right]$calculated for $\mathrm{C}_{8} \mathrm{H}_{7} \mathrm{SN}$ : 149.0293; found: 149.0284 .

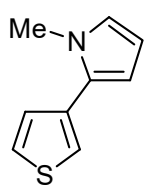

1-Methyl-2-(thiophen-3-yl)-1H-pyrrole (3c): The product was obtained following the general procedure $B$, using 20 mol\% catalyst (Rh-6G $0.02 \mathrm{mmol}, 0.2$ equiv.) in $48 \mathrm{~h}$ of reaction, as a colorless oil in $62 \%$ yield. Once the reaction is finished, the crude product is absorbed on silica gel, the solvent removed under vacuum, and the product purified by flash chromatography (eluent 90/10, pentane/Et ${ }_{2} \mathrm{O}$ ). ${ }^{1} \mathrm{H}-\mathrm{NMR}$ (300 $\left.\mathrm{MHz}, \mathrm{CDCl}_{3}\right)$ ) $7.43-7.33(\mathrm{~m}, 1 \mathrm{H}), 7.21(\mathrm{~s}, 1 \mathrm{H}), 7.20(\mathrm{dd}, J=2.8,1.3 \mathrm{~Hz}, 1 \mathrm{H}), 6.69(\mathrm{~s}, 1 \mathrm{H}), 6.28$ (d, $J=3.5 \mathrm{~Hz}, 1 \mathrm{H}), 6.19(\mathrm{~s}, 1 \mathrm{H}), 3.71(\mathrm{~s}, 3 \mathrm{H}) .{ }^{13} \mathrm{C}-\mathrm{NMR}\left(300 \mathrm{MHz}, \mathrm{CDCl}_{3}\right.$ ) $\delta: 133.9,129.7,128.2$, 125.3, 123.4, 120.7, 108.6, 107.6, 35.3. HMRS: $\left[\mathrm{M}^{+}\right]$calculated $\mathrm{C}_{9} \mathrm{H}_{9} \mathrm{SN}$ : 163. 0450; found: 163.0448 .

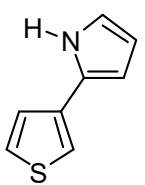

2-(Thiophen-3-yl)-1H-pyrrole (3d): The product was obtained following the general procedure A using 20 mol\% catalyst (Rh-6G $0.02 \mathrm{mmol}, 0.2$ equiv.) in $48 \mathrm{~h}$ of reaction, as a colorless oil in $49 \%$ yield. After the workup, the crude product is absorbed in silica gel, the solvent evaporated under vacuum, and the product purified by flash chromatography (eluent 90/10, pentane/Et $\left.{ }_{2} \mathrm{O}\right) .{ }^{1} \mathrm{H}-\mathrm{NMR}\left(300 \mathrm{MHz}, \mathrm{CDCl}_{3}\right) \delta$ : 8.33 (bs, $1 \mathrm{H}$ ), $7.37-7.32(\mathrm{~m}, 1 \mathrm{H}), 7.25$ (dd, $J=5.0,1.3 \mathrm{~Hz}, 2 \mathrm{H}), 7.17$ (dd, $J=2.9,1.3 \mathrm{~Hz}, 1 \mathrm{H}$ ), $6.85-6.77(\mathrm{~m}, 1 \mathrm{H}), 6.41$ (ddd, $J=4.0,3.1,1.7 \mathrm{~Hz}, 1 \mathrm{H}), 6.32-6.21(\mathrm{~m}, 1 \mathrm{H}) .{ }^{13} \mathrm{C}-\mathrm{NMR}(300 \mathrm{MHz}$, $\left.\mathrm{CDCl}_{3}\right)$ ס: 134.4, 132.1, 126.2, 125.3, 118.0, 116.4, 109.7, 105.9. HMRS: [M+] calculated for $\mathrm{C}_{8} \mathrm{H}_{7} \mathrm{SN}: 149.0293$;found: 149.0290 .

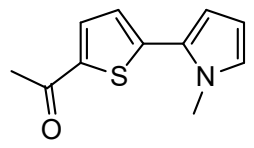

1-(5-(1-Methyl-1H-pyrrol-2-yl)thiophen-2-yl)ethanone (3e): The product was obtained following the general procedure $B$, using $10 \mathrm{~mol} \%$ catalyst (Rh$6 \mathrm{G} 0.01 \mathrm{mmol}, 0.1$ equiv) in $24 \mathrm{~h}$ of reaction, as a brown oil in $96 \%$ yield, after flash chromatography (eluent 85/15 PE/AcOEt). ${ }^{1} \mathrm{H}-\mathrm{NMR}\left(300 \mathrm{MHz}, \mathrm{CDCl}_{3}\right) \delta: 7.62$ (d, J=4.0 Hz, $1 \mathrm{H}), 7.05(\mathrm{~d}, J=4.0 \mathrm{~Hz}, 1 \mathrm{H}), 6.81-6.70(\mathrm{~m}, 1 \mathrm{H}), 6.50(\mathrm{dd}, J=3.8,1.8 \mathrm{~Hz}, 1 \mathrm{H}), 6.18(\mathrm{dd}, J=3.8$, $2.7 \mathrm{~Hz}, 1 \mathrm{H}), 3.81(\mathrm{~s}, 3 \mathrm{H}), 2.55$ (s, 3H). ${ }^{13} \mathrm{C}-\mathrm{NMR}\left(300 \mathrm{MHz}, \mathrm{CDCl}_{3}\right)$ 8: 190.5, 143.9, 141.7, $133.2,126.8,126.1,124.3,111.6,108.6,35.9,26.5$. HMRS: $\left[\mathrm{M}^{+}\right]$calculated for $\mathrm{C}_{11} \mathrm{H}_{11} \mathrm{SON}$ : 205.0555; found: 205.0557. 
$\prod_{S}$

1-(5-(1H-Pyrrol-2-yl)thiophen-2-yl)ethanone(3f): The product was obtained following the general procedure $B$, using 10 mol\% catalyst (Rh-6G $0.01 \mathrm{mmol}$, 0.1 equiv.) in $24 \mathrm{~h}$ of reaction, as a brown oil in $72 \%$ yield, after flash chromatography (eluent 70/30, PE/AcOEt). ${ }^{1} \mathrm{H}-\mathrm{NMR}$ (300 MHz, $\mathrm{CDCl}_{3}$ ) $\delta: 8.69$ (bs, $1 \mathrm{H}$ ), 7.59 (d, $J=4.0 \mathrm{~Hz}, 1 \mathrm{H}$ ), $7.04(\mathrm{~d}, J=4.0 \mathrm{~Hz}, 1 \mathrm{H}), 6.89(\mathrm{td}, J=2.7,1.4 \mathrm{~Hz}, 1 \mathrm{H}), 6.59$ (ddd, $J=3.8,2.6,1.4$ $\mathrm{Hz}, 1 \mathrm{H}), 6.29(\mathrm{dt}, J=3.5,2.6 \mathrm{~Hz}, 1 \mathrm{H}), 6.18-6.16(\mathrm{~m}, 1 \mathrm{H}), 2.53(\mathrm{~s}, 3 \mathrm{H}) .{ }^{13} \mathrm{C}-\mathrm{NMR}(300 \mathrm{MHz}$,

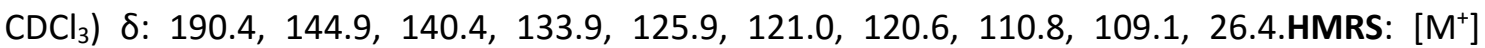
calculated for $\mathrm{C}_{10} \mathrm{H}_{9} \mathrm{SON}$ : 191.0399; found: 191.0398 .

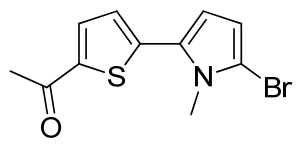

1-(5-(5-Bromo-1-methyl-1H-pyrrol-2-yl)thiophen-2-yl)ethanone (3g): The product was obtained following the general procedure $B$, using $10 \mathrm{~mol} \%$ catalyst ( $\mathrm{Rh}-6 \mathrm{G} 0.01 \mathrm{mmol}, 0.1$ equiv) in $24 \mathrm{~h}$ of reaction, as a yellow solid in 70\% yield, after flash chromatography (eluent 98/2 PE/AcOEt). ${ }^{1} \mathrm{H}-\mathrm{NMR}\left(300 \mathrm{MHz}, \mathrm{CDCl}_{3}\right) \delta$ : $7.62(\mathrm{~d}, J=3.9 \mathrm{~Hz}, 1 \mathrm{H}), 7.02(\mathrm{~d}, J=3.9 \mathrm{~Hz}, 1 \mathrm{H}), 6.44(\mathrm{~d}, J=4.2 \mathrm{~Hz}, 1 \mathrm{H}), 6.24(\mathrm{~d}, J=4.2 \mathrm{~Hz}, 1 \mathrm{H})$, $3.74(\mathrm{~s}, 3 \mathrm{H}), 2.55$ (s, 3H). ${ }^{13} \mathrm{C}-\mathrm{NMR}\left(300 \mathrm{MHz}, \mathrm{CDCl}_{3}\right.$ ) $\delta: 190.5,143.2,142.4,133.0,127.7,125.1$, 111.8, 111.4, 107.0, 34.1, 26.6. HMRS: [ $\left.\mathrm{M}^{+}\right]$calculated for $\mathrm{C}_{11} \mathrm{H}_{10} \mathrm{SONBr}$ : 282.9661; found: 282.9657.<smiles>Cn1cccc1-c1ccc(C=O)s1</smiles>

5-(1-Methyl-1H-pyrrol-2-yl)thiophene-2-carbaldehyde (3h): The product was obtained following the general procedure $B$, using 10 mol\% catalyst (Rh6G $0.01 \mathrm{mmol}, 0.1$ equiv) in $24 \mathrm{~h}$ of reaction, as a yellow oil in $84 \%$ yield, after flash chromatography (eluent 85/15, PE/AcOEt). ${ }^{1} \mathrm{H}-\mathrm{NMR}\left(300 \mathrm{MHz}, \mathrm{CDCl}_{3}\right)$ $\delta: 9.85$ (s, $\left.1 \mathrm{H}\right), 7.77$ $-7.65(\mathrm{~m}, 1 \mathrm{H}), 7.14(\mathrm{t}, J=3.1 \mathrm{~Hz}, 1 \mathrm{H}), 6.86-6.73(\mathrm{~m}, 1 \mathrm{H}), 6.62-6.51(\mathrm{~m}, 1 \mathrm{H}), 6.21$ (ddd, $J=$ 6.5, 2.5, $1.6 \mathrm{~Hz}, 1 \mathrm{H}), 3.83(\mathrm{~s}, 3 \mathrm{H}) .{ }^{13} \mathrm{C}-\mathrm{NMR}\left(300 \mathrm{MHz}, \mathrm{CDCl}_{3}\right) \delta: 182.6,145.3,140.9,137.3$, 126.7, 126.6, 124.2, 112.2, 108.9, 36.0. HMRS: [M+] calculated for $\mathrm{C}_{10} \mathrm{H}_{9} \mathrm{SNO}$ : 191.0399; found: 191.0397.<smiles>O=Cc1ccc(-c2ccc[nH]2)s1</smiles>

5-(1H-Pyrrol-2-yl)thiophene-2-carbaldehyde (3i): The product was obtained following the general procedure $B$, using 10 mol\% catalyst (Rh-6G 0.01 mmol, 0.1 equiv.) in $24 \mathrm{~h}$ of reaction, as a yellow oil in $79 \%$ yield, after flash chromatography (eluent 70/30, PE/AcOEt). ${ }^{1} \mathrm{H}-\mathrm{NMR}\left(300 \mathrm{MHz}, \mathrm{CDCl}_{3}\right) \delta: 9.83(\mathrm{~s}, 1 \mathrm{H}), 8.71$ (s, $1 \mathrm{H}), 7.67(\mathrm{~d}, J=4.0 \mathrm{~Hz}, 1 \mathrm{H}), 7.13(\mathrm{~d}, J=4.0 \mathrm{~Hz}, 1 \mathrm{H}), 6.93(\mathrm{dd}, J=4.0,2.6 \mathrm{~Hz}, 1 \mathrm{H}), 6.77-6.57(\mathrm{~m}$, $1 \mathrm{H}), 6.31(\mathrm{dt}, J=3.4,2.6 \mathrm{~Hz}, 1 \mathrm{H}) .{ }^{13} \mathrm{C}-\mathrm{NMR}\left(300 \mathrm{MHz}, \mathrm{CDCl}_{3}\right) \delta: 182.5,146.2,139.7,138.1$, 125.7, 121.2, 121.1, 111.1, 109.9. HMRS: $\left[\mathrm{M}^{+}\right]$calculated for $\mathrm{C}_{9} \mathrm{H}_{7} \mathrm{SNO}$ : 177.0243; found: 177.0241.<smiles>O=Cc1ccc(-c2cccn2-c2ccccc2)s1</smiles>

5-(1-Phenyl-1H-pyrrol-2-yl)thiophene-2-carbaldehyde (3j): The product was obtained following the general procedure B, using $10 \mathrm{~mol} \%$ catalyst (Rh-6G $0.01 \mathrm{mmol}, 0.1$ equiv.) in $24 \mathrm{~h}$ of reaction, as a colorless oil in $91 \%$ yield, after 
flash chromatography (eluent 90/10, PE/AcOEt). ${ }^{1} \mathrm{H}-\mathrm{NMR}\left(300 \mathrm{MHz}, \mathrm{CDCl}_{3}\right) \delta: 9.74(\mathrm{~s}, 1 \mathrm{H})$, 7.52-7.39 (m, 4H), $7.32-7.27(\mathrm{~m}, 2 \mathrm{H}), 6.95(\mathrm{td}, J=3.1,1.1 \mathrm{~Hz}, 1 \mathrm{H}), 6.70(\mathrm{dt}, J=5.2,2.6 \mathrm{~Hz}$, $1 \mathrm{H}), 6.60(\mathrm{~d}, J=4.0 \mathrm{~Hz}, 1 \mathrm{H}), 6.40-6.32(\mathrm{~m}, 1 \mathrm{H}) .{ }^{13} \mathrm{C}-\mathrm{NMR}\left(300 \mathrm{MHz}, \mathrm{CDCl}_{3}\right) \delta: 182.5,145.3$, 140.8, 139.4, 136.9 (2C), 129.4, 128.5, 127.0, 126.9, 124.5, 113.1, 110.0.HMRS: [M ${ }^{+}$] calculated for $\mathrm{C}_{15} \mathrm{H}_{11}$ NOS: 253.0555; found: 253.0545 .

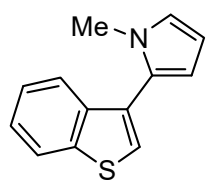

2-(Benzo[b]thiophen-3-yl)-1-methyl-1H-pyrrole (3k): The product was obtained following the general procedure $B$, using 20 mol\% catalyst (Rh-6G $0.02 \mathrm{mmol}, 0.2$ equiv.) in $48 \mathrm{~h}$ of reaction, as a colorless oil in $80 \%$ yield, after lash chromatography (eluent 80/20, pentane/Et 2 ). ${ }^{1} \mathrm{H}-\mathrm{NMR}\left(300 \mathrm{MHz}, \mathrm{CDCl}_{3}\right.$ ) $\delta: ~ 7.96-7.87(\mathrm{~m}, 1 \mathrm{H}), 7.82-7.73(\mathrm{~m}, 1 \mathrm{H}), 7.44-7.36(\mathrm{~m}, 2 \mathrm{H}), 7.35(\mathrm{~s}, 1 \mathrm{H}), 6.82-6.80(\mathrm{~m}, 1 \mathrm{H})$, $6.38-6.33(\mathrm{~m}, 1 \mathrm{H}), 6.31-6.29(\mathrm{~m}, 1 \mathrm{H}), 3.59(\mathrm{~s}, 3 \mathrm{H}) .{ }^{13} \mathrm{C}-\mathrm{NMR}\left(300 \mathrm{MHz}, \mathrm{CDCl}_{3}\right) \delta: 139.8,139.1$, $129.0,127.6,124.8,124.5,124.4,123.5,123.2,122.7,109.9,107.8,34.7$. HMRS: $\left[\mathrm{M}^{+}\right]$ calculated for $\mathrm{C}_{13} \mathrm{H}_{11} \mathrm{SN}$ : 213.0606; found: 213.0596 .<smiles>Cn1cccc1-c1ccc(C=O)o1</smiles>

5-(1-Methyl-1H-pyrrol-2-yl)furan-2-carbaldehyde (3l): The product was obtained following the general procedure $B$, using 10 mol\% catalyst (Rh-6G $0.01 \mathrm{mmol}, 0.1$ equiv) in $24 \mathrm{~h}$ of reaction, as a colorless oil in $81 \%$ yield, after flash chromatography (eluent 85/15, pentane/Et $\left.{ }_{2} \mathrm{O}\right) .{ }^{1} \mathrm{H}-\mathrm{NMR}\left(300 \mathrm{MHz}, \mathrm{CDCl}_{3}\right) \delta: 9.55(\mathrm{~s}, 1 \mathrm{H})$, $7.28(\mathrm{~d}, J=3.8 \mathrm{~Hz}, 1 \mathrm{H}), 6.77-6.72(\mathrm{~m}, 1 \mathrm{H}), 6.69(\mathrm{dd}, J=3.9,1.7 \mathrm{~Hz}, 1 \mathrm{H}), 6.53(\mathrm{~d}, J=3.8 \mathrm{~Hz}, 1 \mathrm{H})$, $6.19(\mathrm{dd}, J=3.8,2.6 \mathrm{~Hz}, 1 \mathrm{H}), 3.91(\mathrm{~s}, 3 \mathrm{H}) .{ }^{13} \mathrm{C}-\mathrm{NMR}\left(300 \mathrm{MHz}, \mathrm{CDCl}_{3}\right) \delta: 176.3,154.1,150.9$, 127.0, 123.1, 112.5, 108.9 (2C), 106.8, 36.6. HMRS: [M+] calculated for $\mathrm{C}_{10} \mathrm{H}_{9} \mathrm{NO}_{2}:$ : 175.0627; found: 175.0630 .

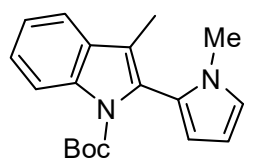

tert-Butyl 3-methyl-2-(1-methyl-1H-pyrrol-2-yl)-1H-indole-1-carboxylate $(3 \mathrm{~m})$ : The product was obtained following the general procedure $B$, using 20 mol\% catalyst (Rh-6G $0.02 \mathrm{mmol}, 0.2$ equiv) in $48 \mathrm{~h}$ of reaction, as a white solid in $49 \%$ yield, after flash chromatography (eluent 92/8, pentane/Et $\left.{ }_{2} \mathrm{O}\right) .{ }^{1} \mathrm{H}-\mathrm{NMR}(300 \mathrm{MHz}$, $\left.\mathrm{CDCl}_{3}\right) \delta: 8.25(\mathrm{~d}, J=8.3 \mathrm{~Hz}, 1 \mathrm{H}), 7.54(\mathrm{dd}, J=7.7,0.6 \mathrm{~Hz}, 1 \mathrm{H}), 7.40-7.33(\mathrm{~m}, 1 \mathrm{H}), 7.32-7.26$ (m, 1H), $6.73(\mathrm{bs}, 1 \mathrm{H}), 6.19(\mathrm{~d}, J=2.2 \mathrm{~Hz}, 1 \mathrm{H}), 6.13(\mathrm{~d}, J=3.4 \mathrm{~Hz}, 1 \mathrm{H}), 3.41(\mathrm{~s}, 3 \mathrm{H}), 2.13(\mathrm{~s}, 3 \mathrm{H})$, 1.37 (s, 10H). ${ }^{13} \mathrm{C}-\mathrm{NMR}\left(300 \mathrm{MHz}, \mathrm{CDCl}_{3}\right) \delta: 150.2,136.5,130.0,127.3,125.0,124.8,122.5$, 122.1, 119.9, 188.9, 115.5, 110.6, 107.4, 82.5, 34.17, 27.9, 9.37.HMRS: [M+H $\left.{ }^{+}\right]$calculated for $\mathrm{C}_{19} \mathrm{H}_{23} \mathrm{~N}_{2} \mathrm{O}_{2}: 311.1754$; found: 311.1755 .

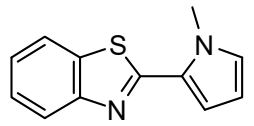

2-(1-Methyl-1H-pyrrol-2-yl)benzo[d]thiazole (3n): The product was obtained following the general procedure A, using 20 mol\% catalyst (Rh-6G $0.02 \mathrm{mmol}$, 0.2 equiv.) in $48 \mathrm{~h}$ of reaction, as a brown oil in $87 \%$ yield, after flash chromatography (eluent 95/5, PE/AcOEt). ${ }^{1} \mathrm{H}-\mathrm{NMR}\left(300 \mathrm{MHz}, \mathrm{CDCl}_{3}\right) \delta: 7.98-7.92(\mathrm{~m}, 1 \mathrm{H})$, $7.82(\mathrm{dd}, J=7.9,0.6 \mathrm{~Hz}, 1 \mathrm{H}), 7.46-7.40(\mathrm{~m}, 1 \mathrm{H}), 7.31(\mathrm{td}, J=7.8,1.1 \mathrm{~Hz}, 1 \mathrm{H}), 6.83(\mathrm{dt}, J=5.0$, 
$1.7 \mathrm{~Hz}, 1 \mathrm{H}), 6.21(\mathrm{dd}, J=3.8,2.6 \mathrm{~Hz}, 1 \mathrm{H}), 4.15(\mathrm{~s}, 1 \mathrm{H}) .{ }^{13} \mathrm{C}-\mathrm{NMR}\left(300 \mathrm{MHz}, \mathrm{CDCl}_{3}\right) \delta: 160.5$, $154.3,133.9,128.0,126.5,125.9,124.5,122.5,121.1,114.9,108.8,37.2$. HMRS: $\left[\mathrm{M}+\mathrm{H}^{+}\right]$ calculated for $\mathrm{C}_{12} \mathrm{H}_{10} \mathrm{~N}_{2} \mathrm{~S}: 214.0565$; found: 214.0556 .

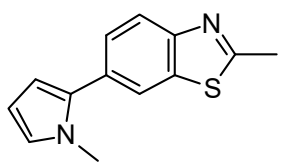

2-Methyl-6-(1-methyl-1H-pyrrol-2-yl)benzo[d]thiazole (30): The product was obtained following the general procedure $A$, using $20 \mathrm{~mol} \%$ catalyst (Rh-6G 0.02 mmol, 0.2 equiv.) in $48 \mathrm{~h}$ of reaction, as a brown oil in $51 \%$ yield, after flash chromatography (eluent 90/10, PE/AcOEt). ${ }^{1} \mathrm{H}-\mathrm{NMR}$ (300 $\mathrm{MHz}, \mathrm{CDCl}_{3}$ ) $\delta: 7.97(\mathrm{~d}, J=1.3 \mathrm{~Hz}, 1 \mathrm{H}$ ), $7.83(\mathrm{dd}, J=8.3,0.5 \mathrm{~Hz}, 1 \mathrm{H}$ ), 7.42 (dd, $J=8.3,1.7 \mathrm{~Hz}$, $1 \mathrm{H}), 6.76(\mathrm{dd}, J=2.5,2.0 \mathrm{~Hz}, 1 \mathrm{H}), 6.30(\mathrm{dd}, J=3.6,1.8 \mathrm{~Hz}, 1 \mathrm{H}), 6.24(\mathrm{dd}, J=3.6,2.7 \mathrm{~Hz}, 1 \mathrm{H})$,

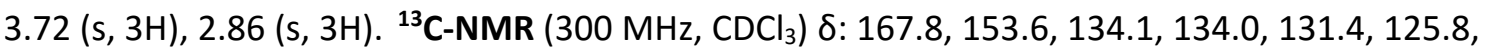
124.0, 121.8, 121.3, 109.1, 107.9, 35.2, 20.2. HMRS: $\left[\mathrm{M}+\mathrm{H}^{+}\right]$calculated for $\mathrm{C}_{13} \mathrm{H}_{12} \mathrm{~N}_{2} \mathrm{~S}$ : 228.0721; found: 228.0712 .

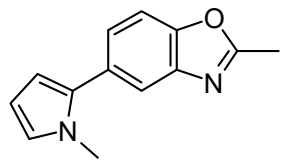

2-Methyl-5-(1-methyl-1H-pyrrol-2-yl)benzo[d]oxazole (3p): The product was obtained following the general procedure $A$, using $20 \mathrm{~mol} \%$ catalyst (Rh-6G $0.02 \mathrm{mmol}, 0.2$ equiv.) in $48 \mathrm{~h}$ of reaction, as a white solid in $79 \%$ yield, after flash chromatography (eluent 90/10, PE/AcOEt). ${ }^{1} \mathrm{H}-\mathrm{NMR}$ (300

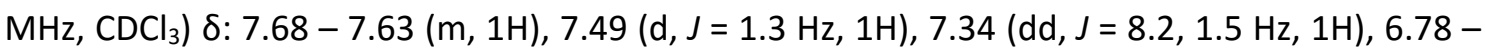
$6.71(\mathrm{~m}, 1 \mathrm{H}), 6.26(\mathrm{dd}, J=3.6,1.8 \mathrm{~Hz}, 1 \mathrm{H}), 6.23-6.20(\mathrm{~m}, 1 \mathrm{H}), 3.68(\mathrm{~s}, 3 \mathrm{H}), 2.66(\mathrm{~s}, 3 \mathrm{H}) .{ }^{13} \mathrm{C}-$ NMR (300 MHz, $\left.\mathrm{CDCl}_{3}\right)$ ) $: 164.2,151.1,140.4,134.2,130.1,125.3,123.8,119.0,110.3,109.1$, 107.9, 35.1, 14.6. HMRS: $\left[\mathrm{M}+\mathrm{H}^{+}\right]$calculated for $\mathrm{C}_{13} \mathrm{H}_{12} \mathrm{~N}_{2} \mathrm{O}: 212.0944$; found: 212.0946 .

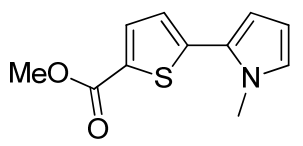

Methyl 5-(1-methyl-1H-pyrrol-2-yl)thiophene-2-carboxylate (3q): The product was obtained following the general procedure $\mathrm{B}$, using $10 \mathrm{~mol} \%$ catalyst (Rh-6G $0.01 \mathrm{mmol}, 0.1$ equiv) in $48 \mathrm{~h}$ of reaction, as a uncolored oil in $57 \%$ yield, after flash chromatography (eluent 96/4 PE/AcOEt). ${ }^{1} \mathrm{H}-\mathrm{NMR}$ (300 MHz, $\mathrm{CDCl}_{3}$ ) $\delta: 7.74(\mathrm{~d}, J=3.9 \mathrm{~Hz}, 1 \mathrm{H}), 7.01(\mathrm{~d}, J=3.9 \mathrm{~Hz}, 1 \mathrm{H}), 6.80-6.70(\mathrm{~m}, 1 \mathrm{H}), 6.46$ (dd, $J=3.8,1.8 \mathrm{~Hz}$, $1 \mathrm{H}), 6.18(\mathrm{dd}, J=3.7,2.7 \mathrm{~Hz}, 1 \mathrm{H}), 3.89(\mathrm{~s}, 3 \mathrm{H}), 3.79(\mathrm{~s}, 3 \mathrm{H}) .{ }^{13} \mathrm{C}-\mathrm{NMR}\left(300 \mathrm{MHz}, \mathrm{CDCl}_{3}\right) \delta: 162.7$, 142.2, 133.9, 130.8, 126.6, 125.6, 124.3, 111.2, 108.4, 52.1, 35.6. HMRS: [M+] calculated for $\mathrm{C}_{11} \mathrm{H}_{11} \mathrm{SO}_{2} \mathrm{~N}$ : 221.0505; found: 221.0508 .

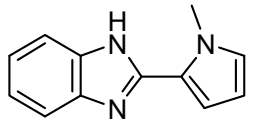

2-(1-Methyl-1H-pyrrol-2-yl)-1H-benzo[d]imidazole (3r): The product was obtained following the general procedure A, using 20 mol\% catalyst (Rh-6G $0.02 \mathrm{mmol}, 0.2$ equiv.) in $48 \mathrm{~h}$ of reaction, as a white solid in $84 \%$ yield, after flash chromatography (eluent 80/20, PE/AcOEt). ${ }^{1} \mathrm{H}-\mathrm{NMR}$ (300 MHz, DMSO-d $\left._{6}\right) \delta: 7.84-7.74$ $(\mathrm{m}, 2 \mathrm{H}), 7.56-7.46(\mathrm{~m}, 2 \mathrm{H}), 7.37-7.31(\mathrm{~m}, 1 \mathrm{H}), 7.21(\mathrm{dd}, J=4.0,1.7 \mathrm{~Hz}, 1 \mathrm{H}), 6.35(\mathrm{dd}, J=4.0$, $2.5 \mathrm{~Hz}, 1 \mathrm{H}), 4.09$ (s, 1H). ${ }^{13} \mathrm{C}-\mathrm{NMR}\left(300 \mathrm{MHz}, \mathrm{DMSO}-\mathrm{d}_{6}\right) \delta: 142.1,131.7,131.3,125.2,117.7$, 116.7, 113.5, 109.5, 35.9. HMRS: [ $\left.\mathrm{M}^{+}\right]$calculated for $\mathrm{C}_{12} \mathrm{H}_{11} \mathrm{~N}_{3}$ : 197.0947; found: 197.0937 . 


\section{Fluorescence quenching experiments:}

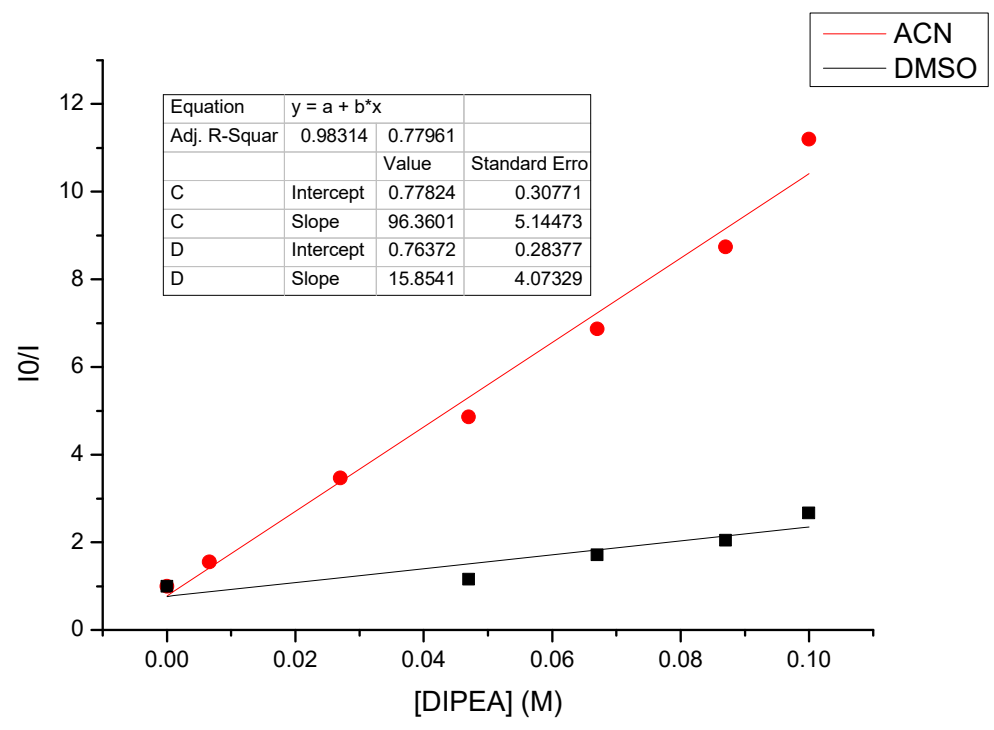

Figure S2: Stern-Volmer quenching plot of Rh-6G in the presence of DIPEA in ACN or DMSO $\left(K_{\mathrm{q}, \mathrm{ACN}}=23.3 \times 10^{9} \mathrm{M}^{-1} \mathrm{~s}^{-1} ; K_{\mathrm{q}, \mathrm{DMSO}}=3.8 \times 10^{9} \mathrm{M}^{-1} \mathrm{~s}^{-1}\right)$.

\section{Cyclic voltammetry:}

Electrochemical studies were performed in $\mathrm{ACN}$ containing $0.1 \mathrm{M}$ tetra- $n$-butylammonium tetrafluoroborate using ferrocene/ferrocenium $\left(\mathrm{Fc} / \mathrm{Fc}^{+}\right)$as an internal reference. A glassy carbon electrode (working electrode), platinum wire counter electrode, and Ag quasireference electrode were employed. Spectroelectrochemical studies were carried out in an optically transparent thin layer electrochemical cell (OTTLE).

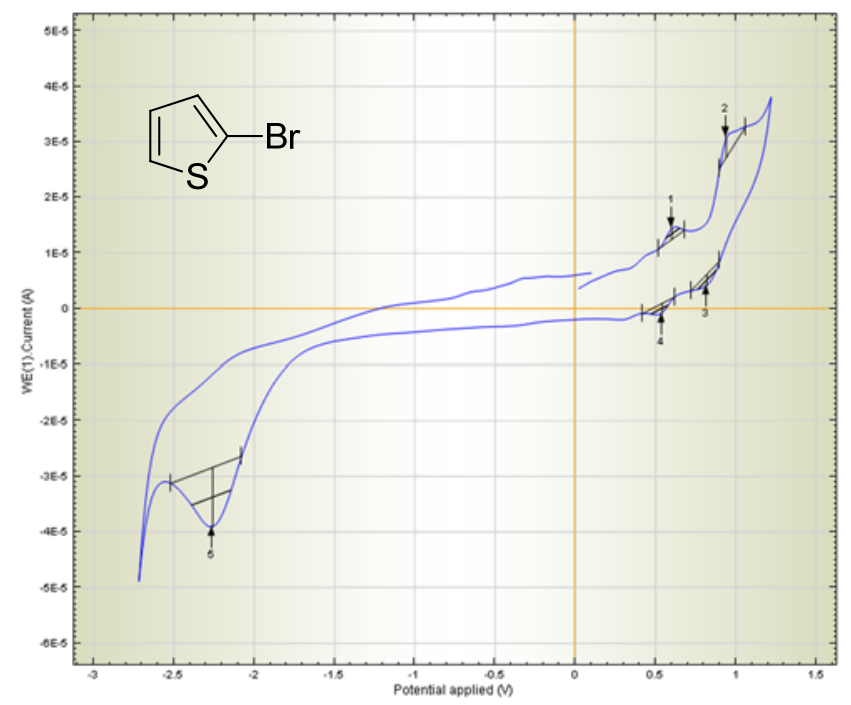

$E_{\text {red }}=-2.4$ eV vs SCE 


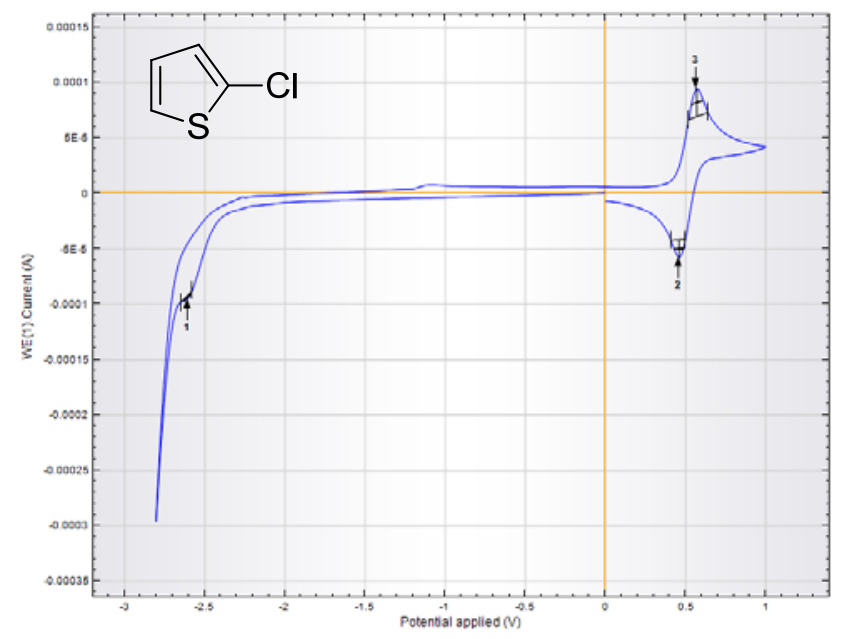

$E_{\text {red }}=-2.7$ eV vs SCE 
5. NMR spectra for compounds 3a-3r:

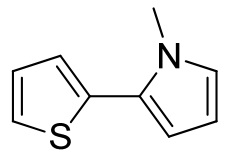

$3 a$

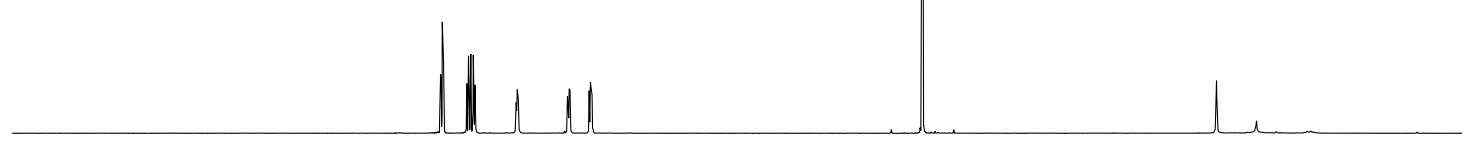

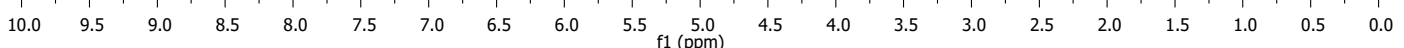



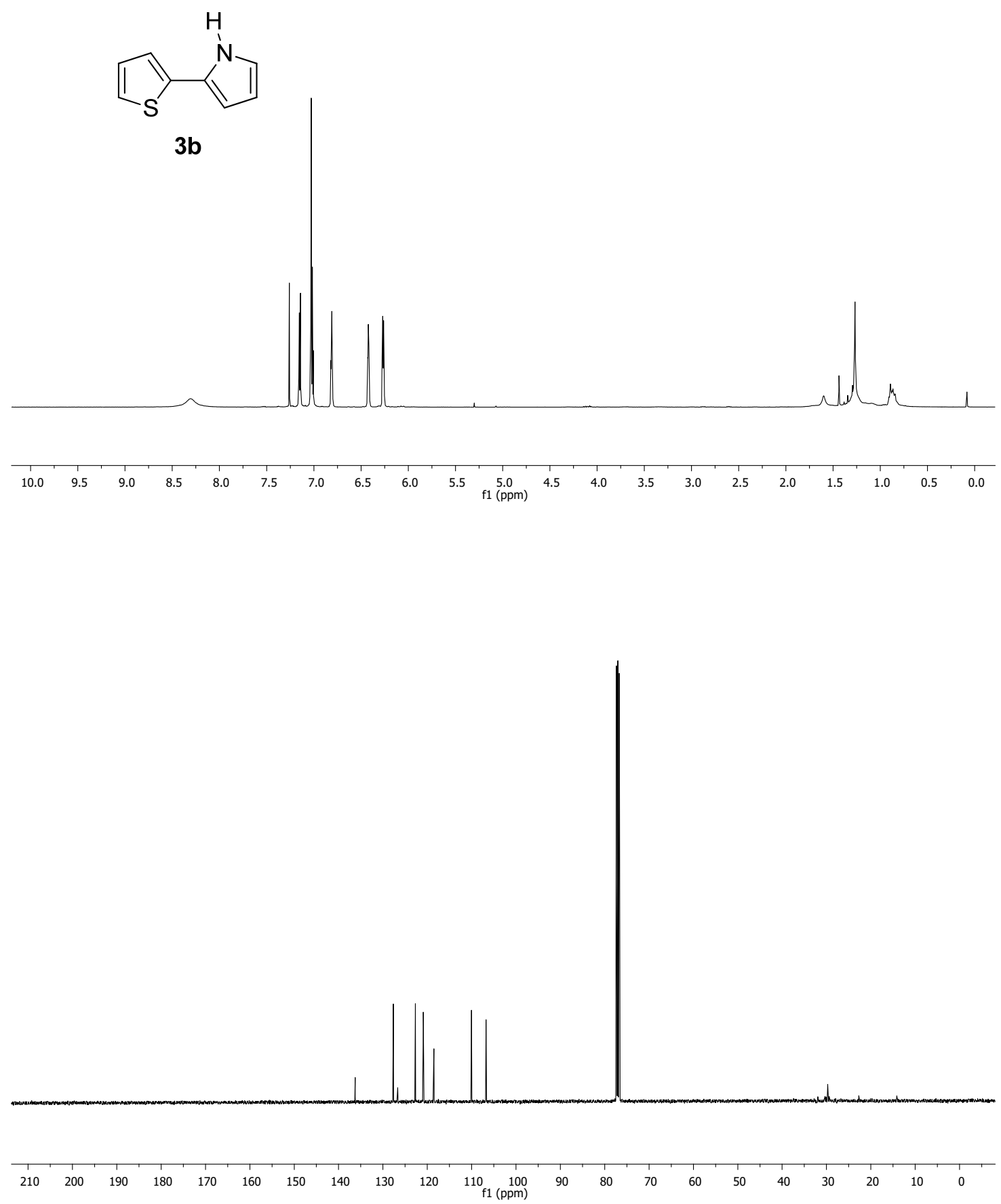
<smiles>Cn1cccc1-c1ccsc1</smiles>

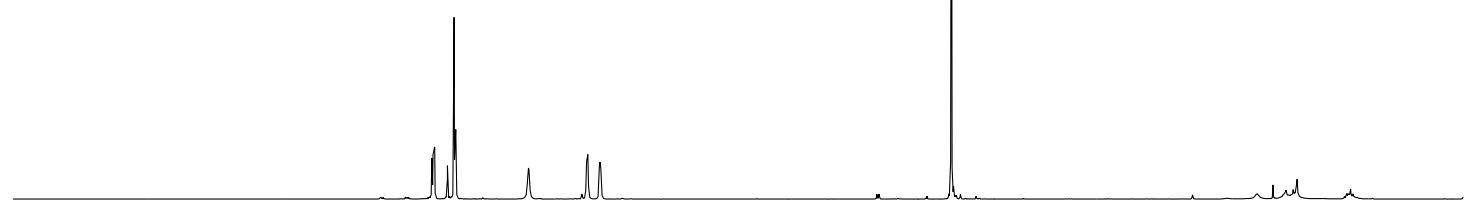

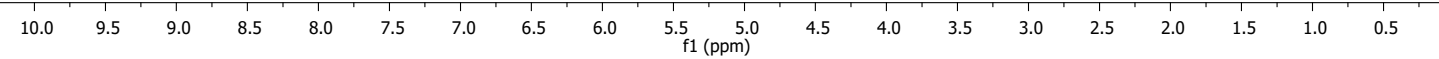

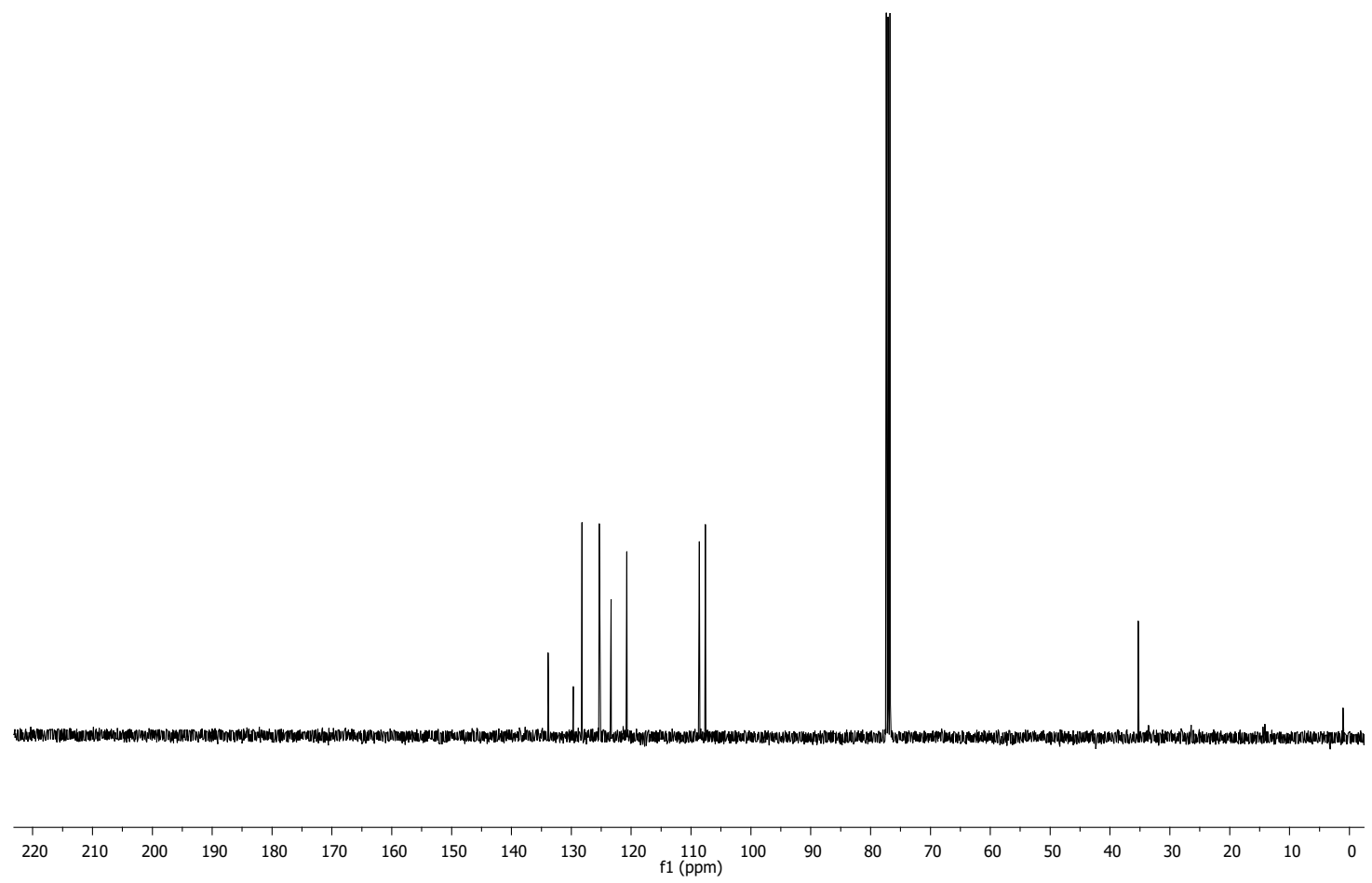


<smiles>c1c[nH]c(-c2ccsc2)c1</smiles>
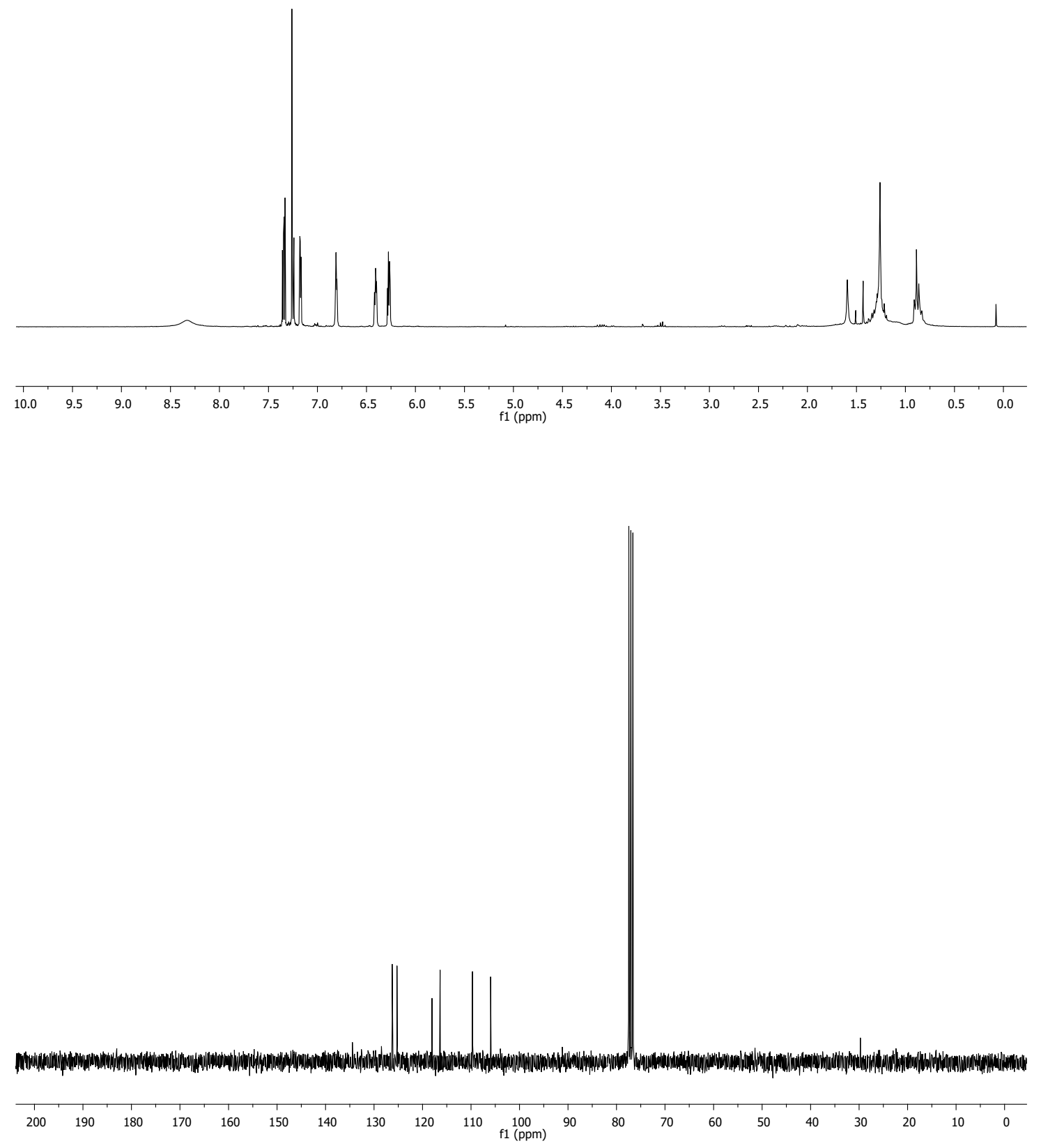


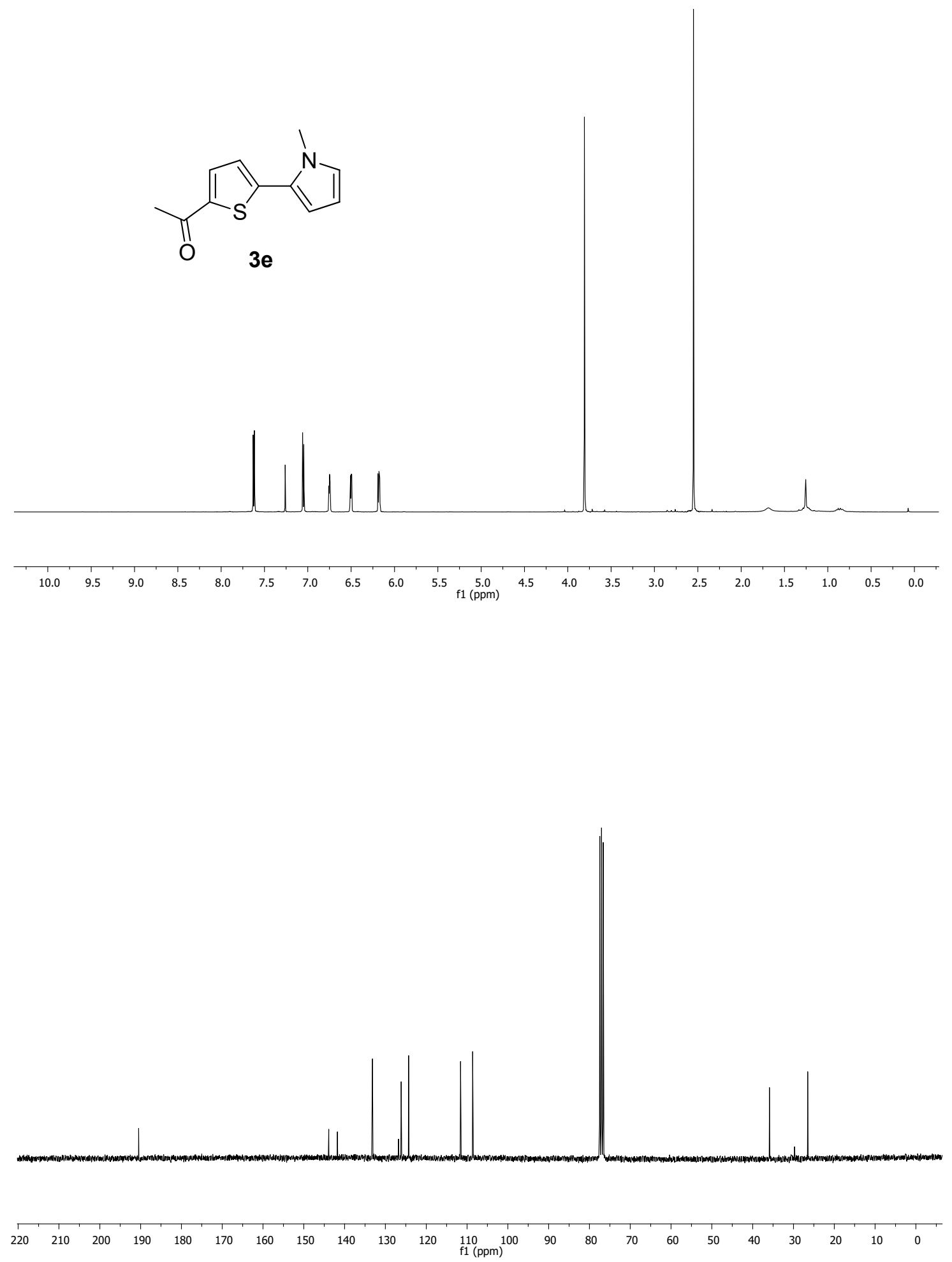




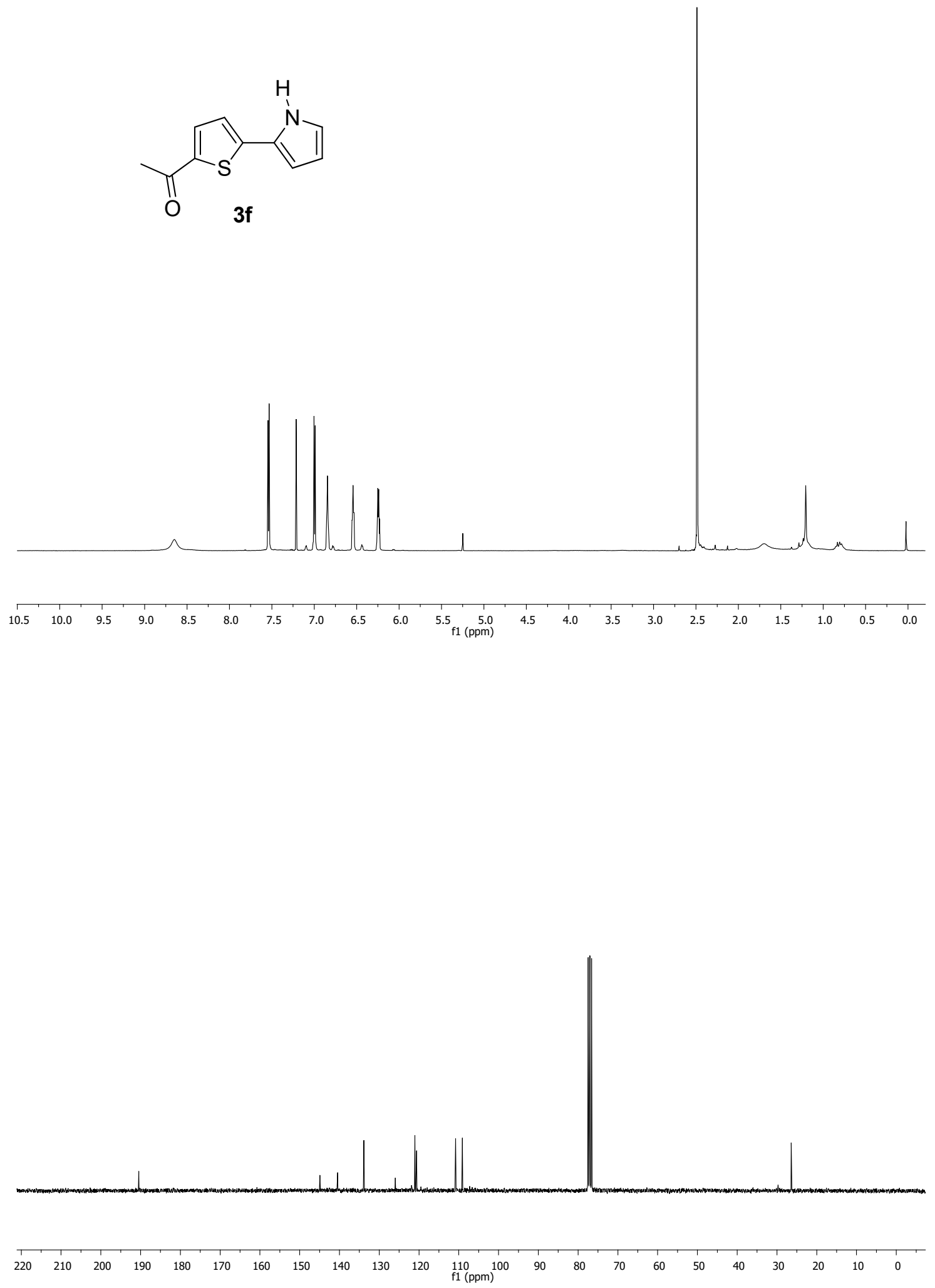



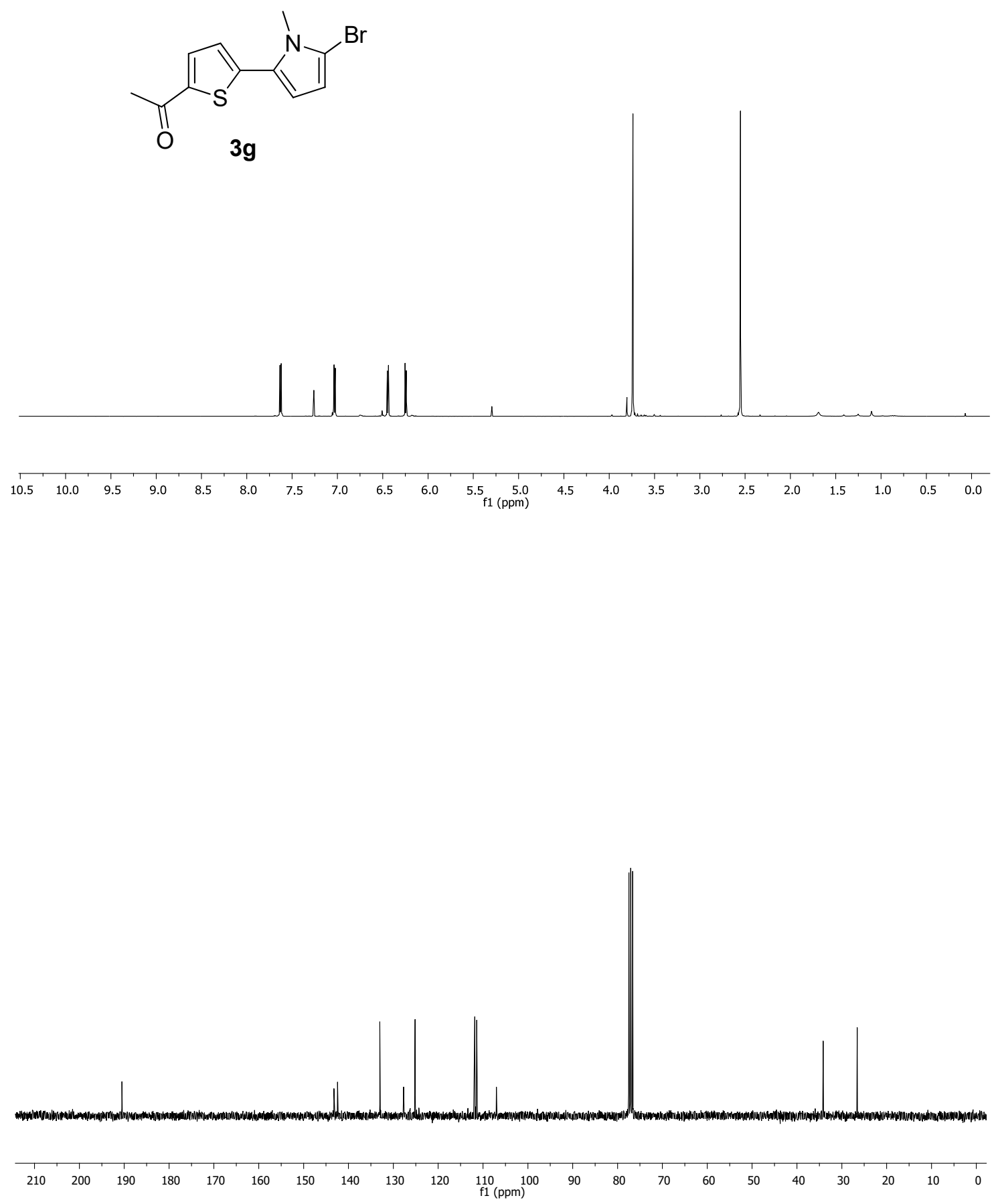
<smiles>Cn1cccc1-c1ccc(C=O)s1</smiles>
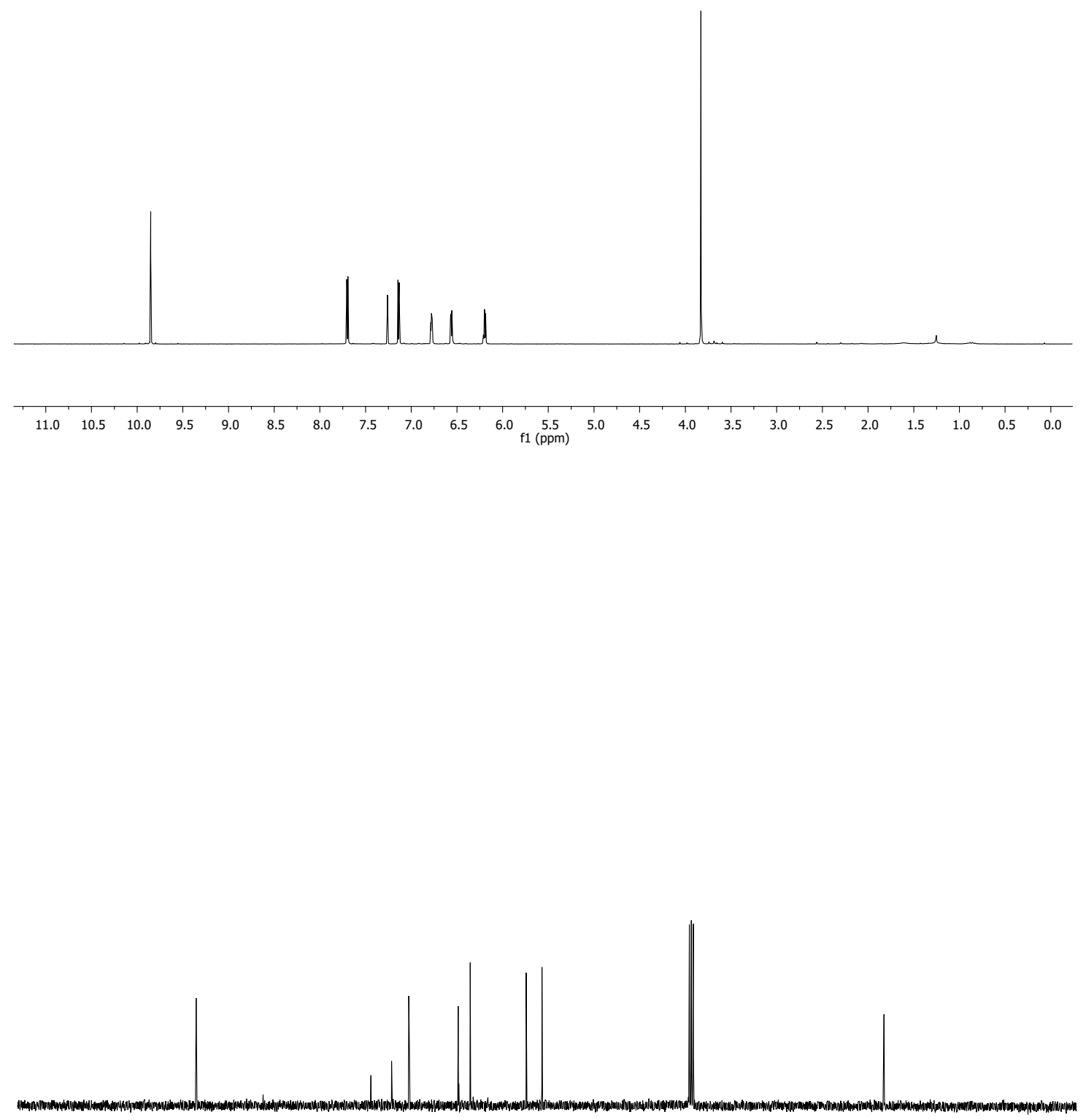

\begin{tabular}{rllllllllllllllllllllll}
\hline 220 & 210 & 200 & 190 & 180 & 170 & 160 & 150 & 140 & 130 & 120 & $\underset{f 1}{110}(\mathrm{ppm})$ & 100 & 90 & 80 & 70 & 60 & 50 & 40 & 30 & 20 & 10 & 0
\end{tabular} 
<smiles>O=Cc1ccc(-c2ccc[nH]2)s1</smiles>

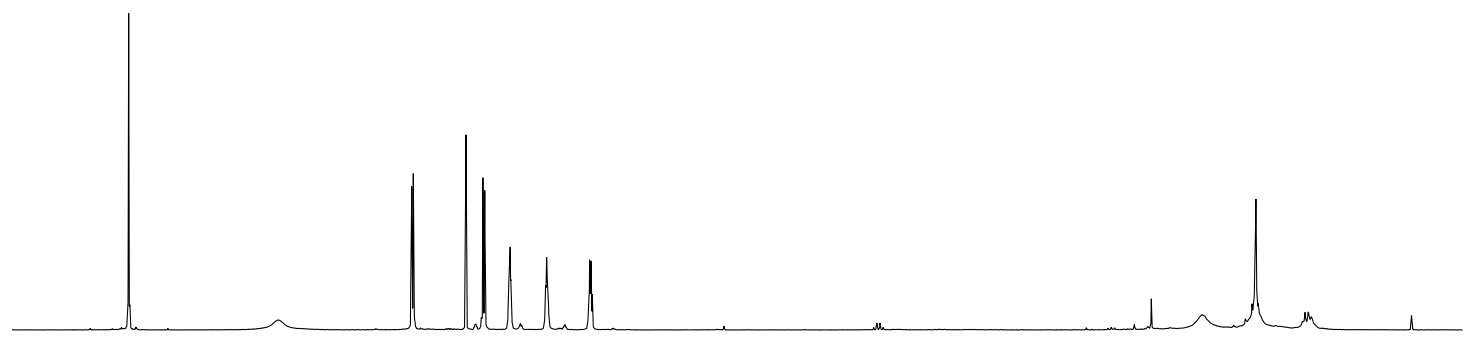

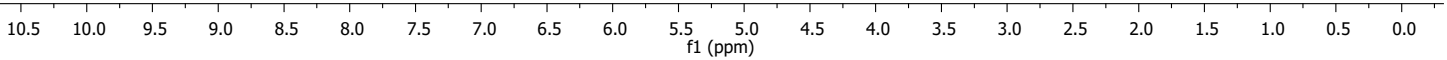

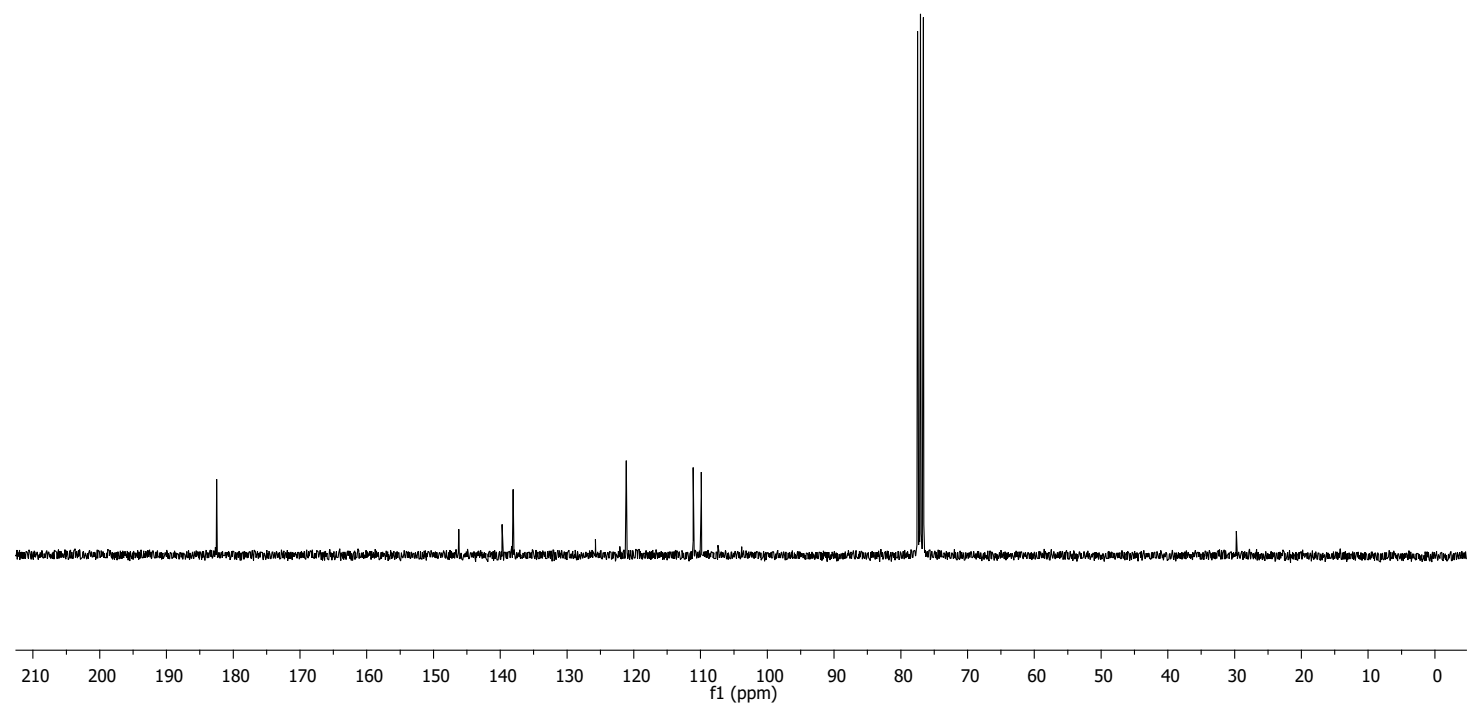



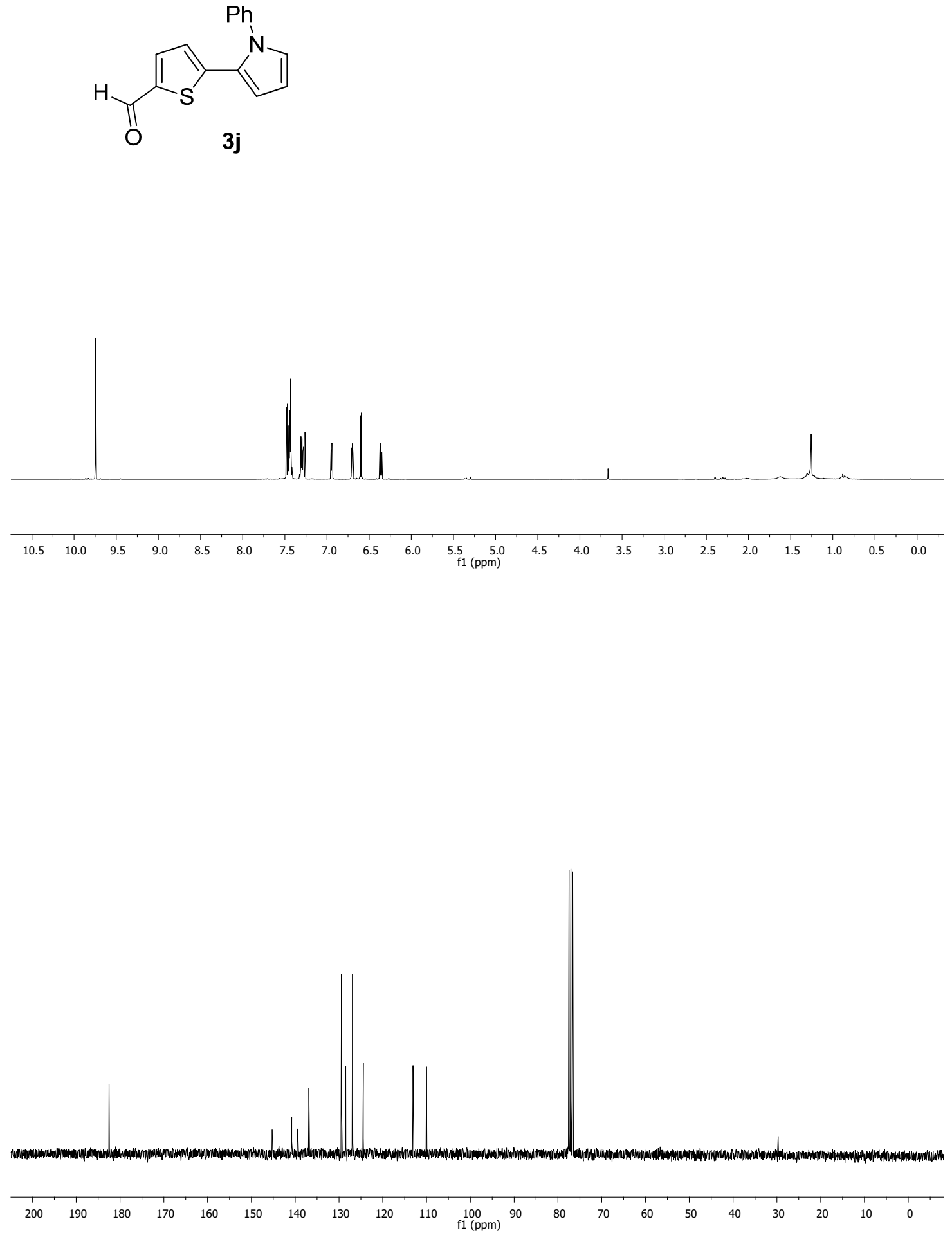

S19 

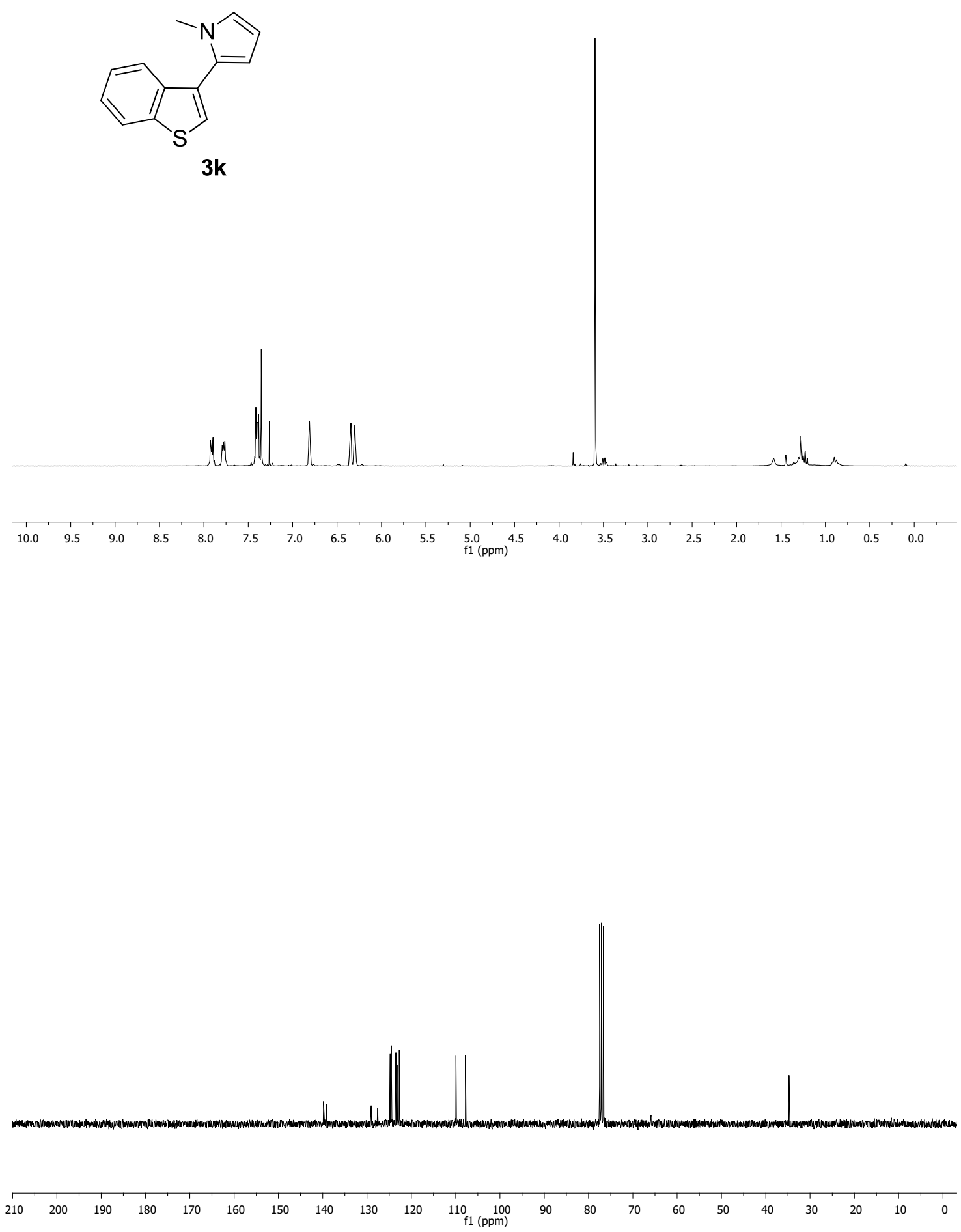
<smiles>Cn1cccc1-c1ccc(C=O)o1</smiles>
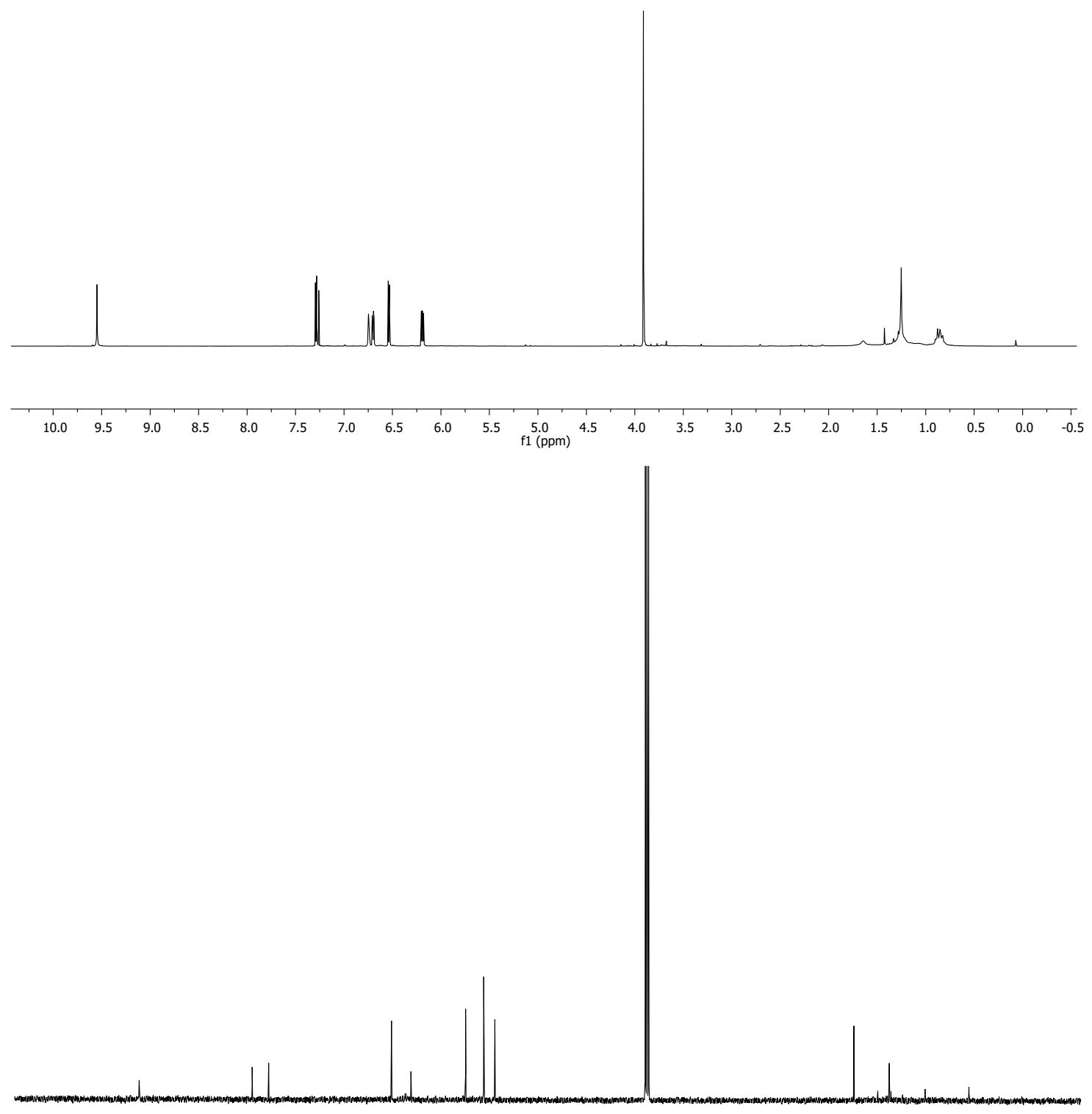

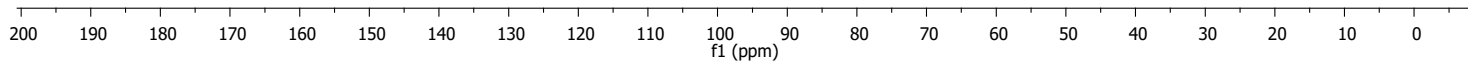



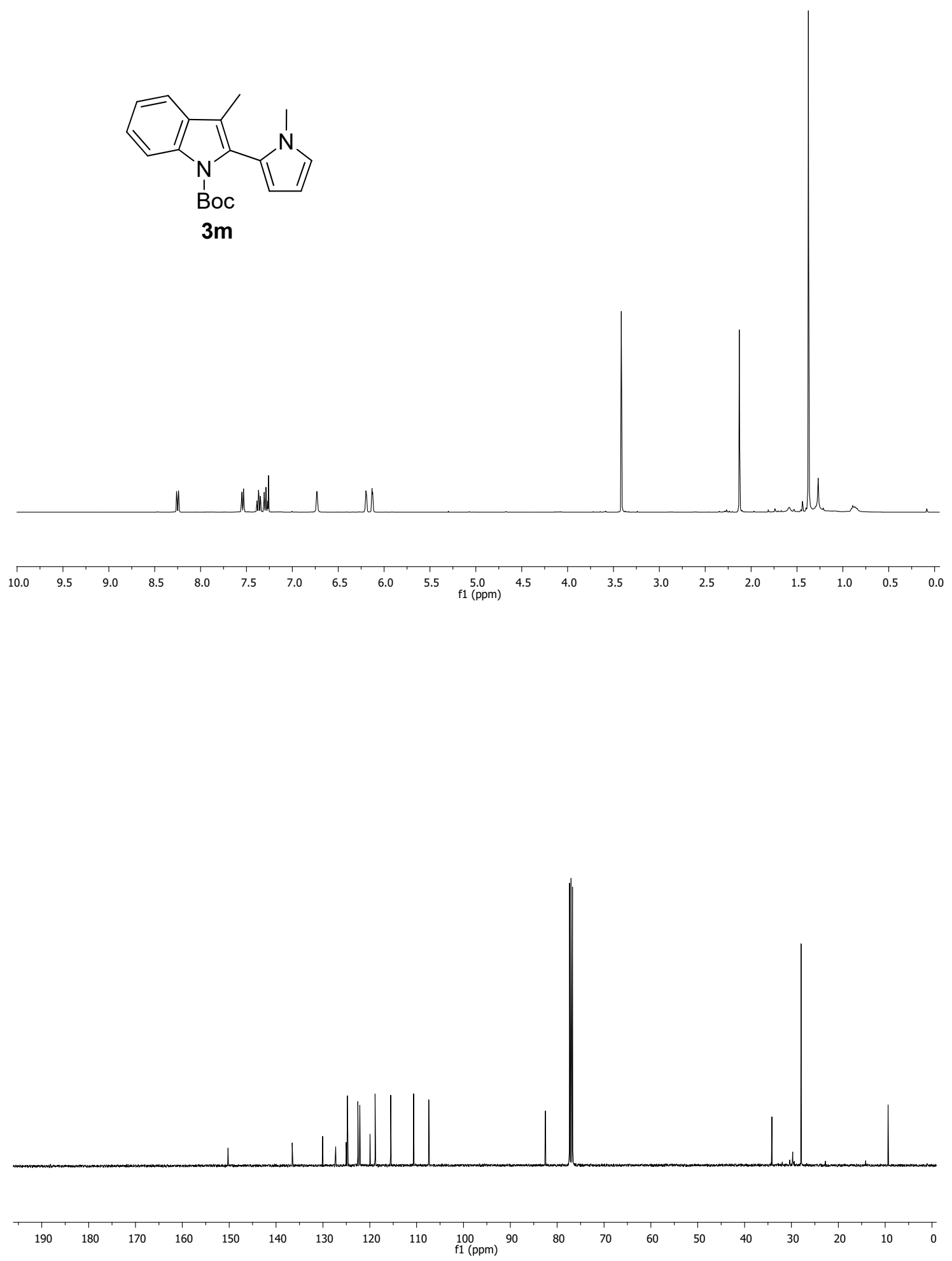

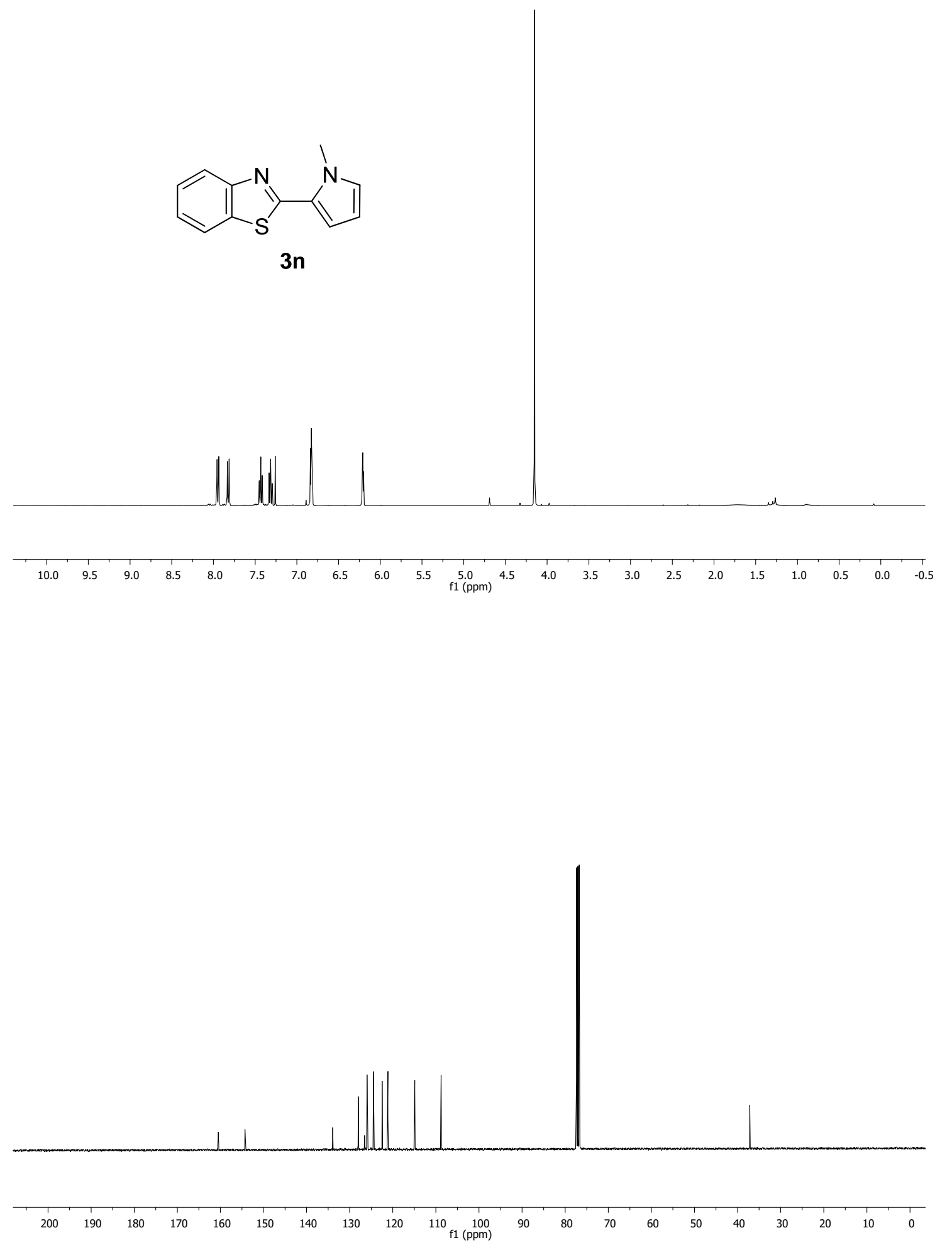


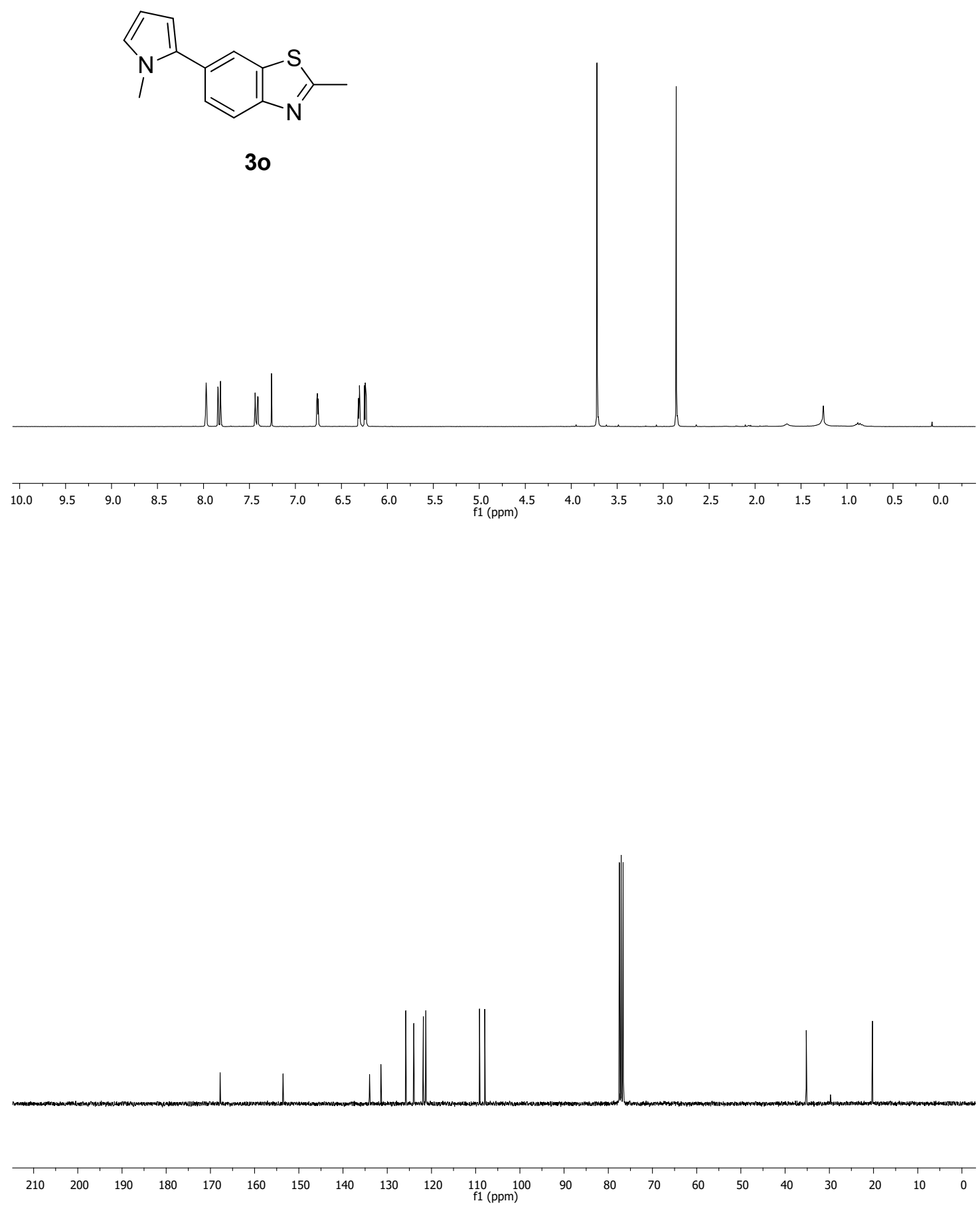



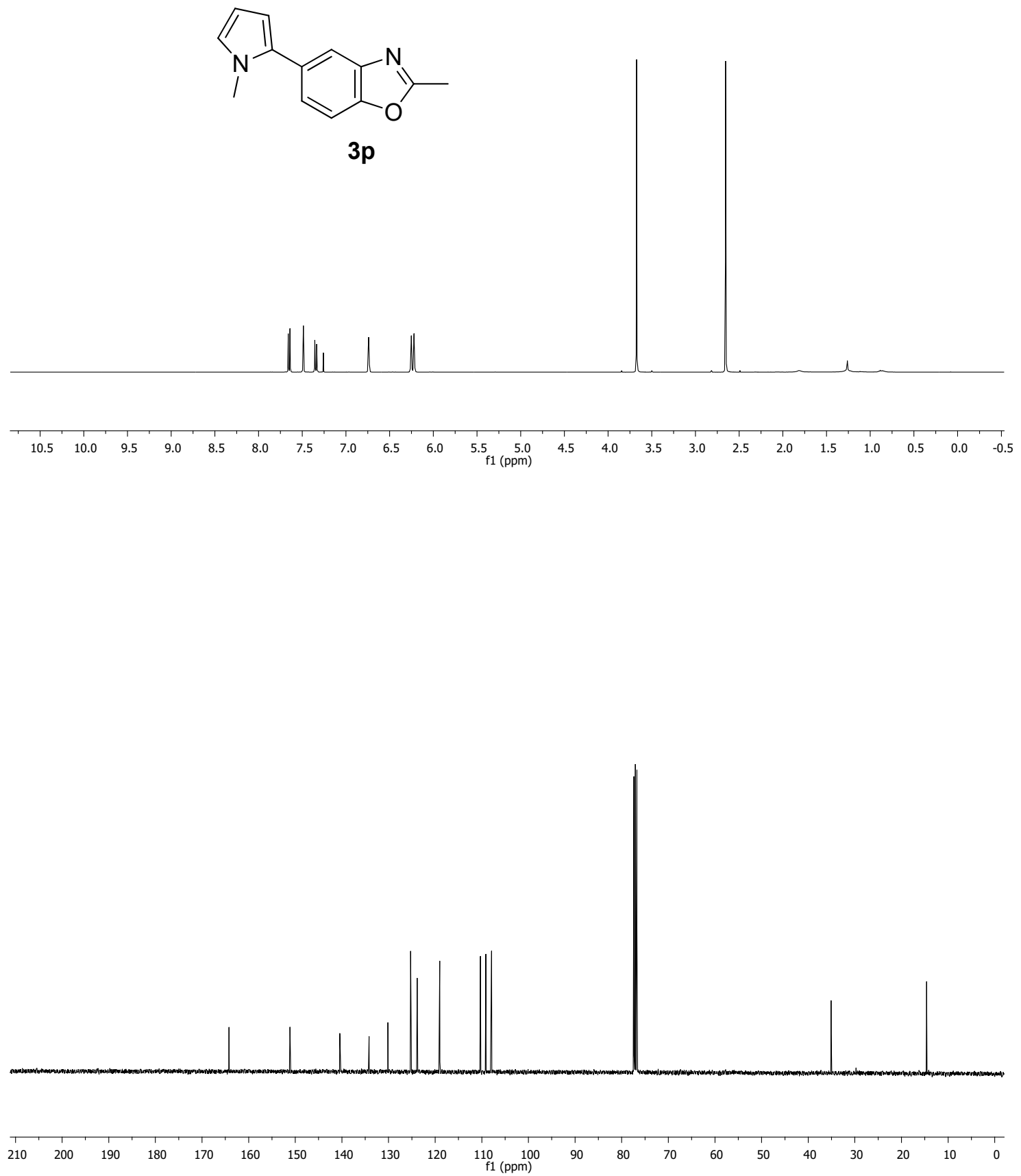

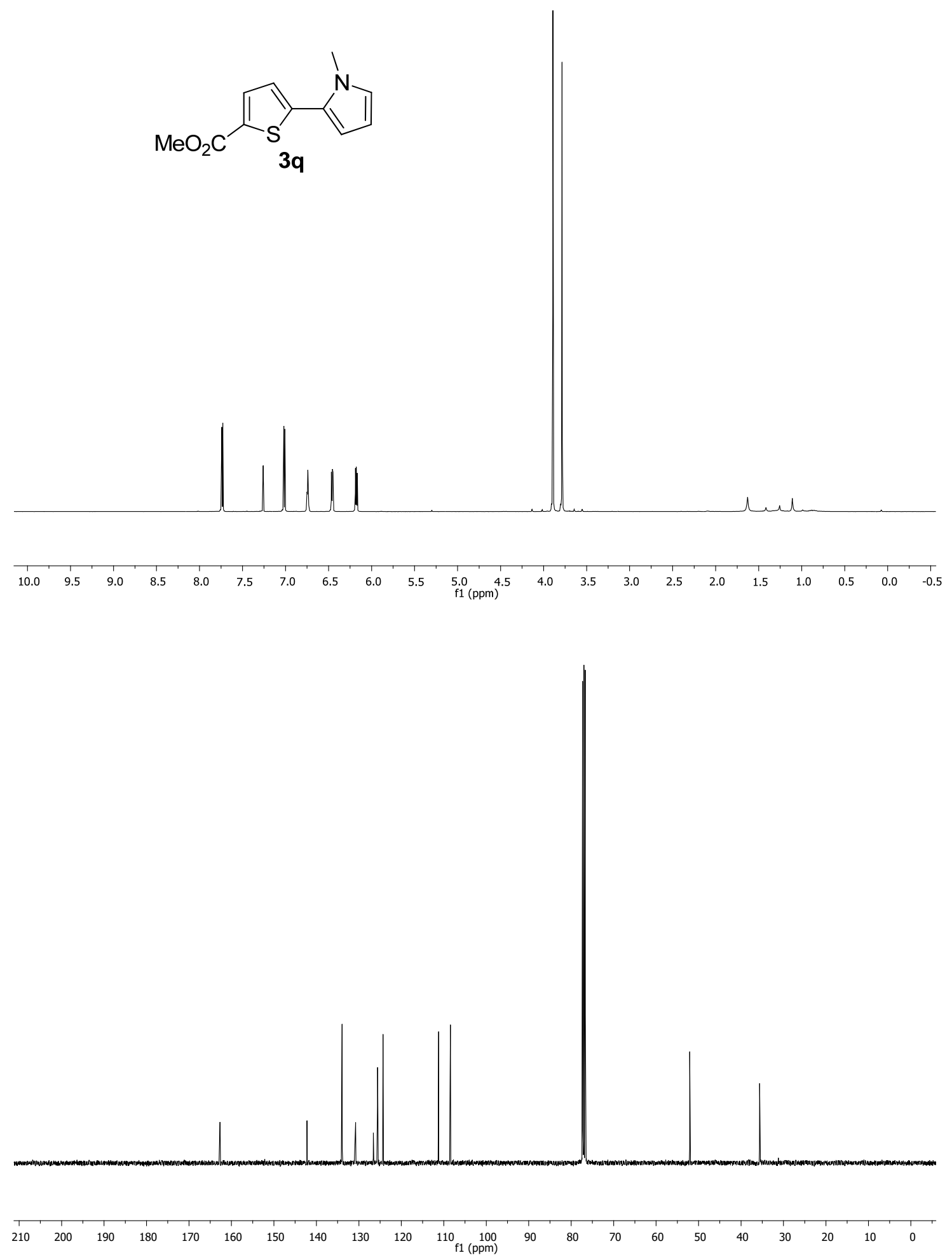

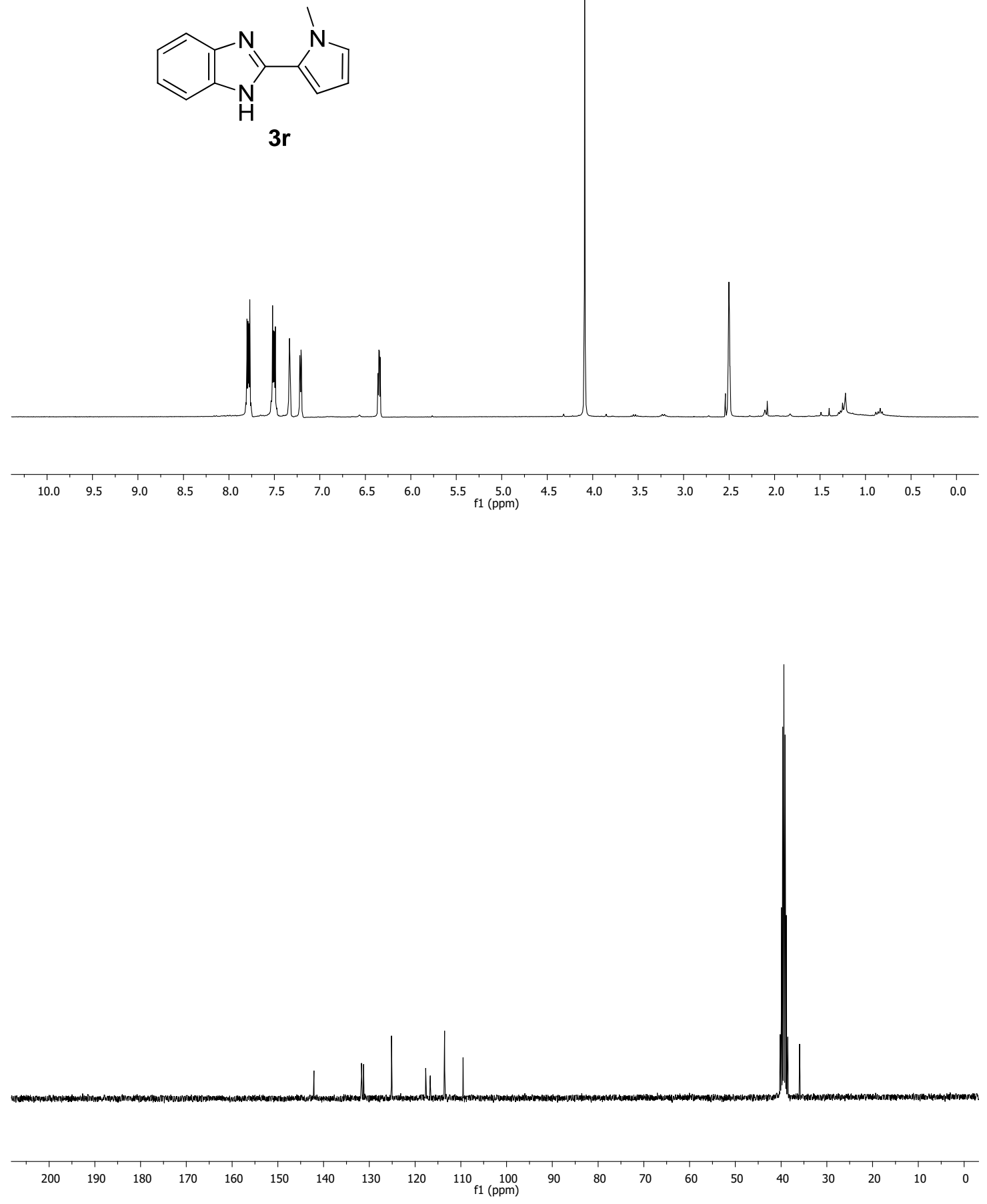

S27 


\section{References:}

(1) (a) Gottlieb, H. E.; Kotlyar, V.; Nudelman, A. J. Org. Chem. 1997, 62, 7512-7515; (b) Fulmer, G. R.; Miller, A. J. M.; Sherden, N. H.; Gottlieb, H. E.; Nudelman, A.; Stoltz, B. M.; Bercaw, J. E.; Goldberg, K. I. Organometallics 2010, 29, 2176-2179.

(2) In some cases, higher catalyst loadings were used as indicated in each case.

(3) Belkessam, F.; Mohand, A.; Soulé, J.-F.; Elias, A.; Doucet, H. Beilstein J. Org. Chem. 2014, 10, 2912-2919. 